\title{
Jet timing
}

\author{
Wen Han Chiu, ${ }^{a}$ Zhen Liu, ${ }^{b}$ Matthew Low ${ }^{c}$ and Lian-Tao Wang ${ }^{a, d, e}$ \\ ${ }^{a}$ Department of Physics, University of Chicago, \\ 5720 South Ellis Avenue, Chicago, IL 60637, U.S.A. \\ ${ }^{b}$ School of Physics and Astronomy, University of Minnesota, \\ 116 Church Street S.E., Minneapolis, MN 55455, U.S.A. \\ ${ }^{c}$ Theoretical Physics Department, Fermilab, \\ P.O. Box 500, Batavia, IL 60510, U.S.A. \\ ${ }^{d}$ Enrico Fermi Institute, University of Chicago, \\ 933 East 56th Street, Chicago, IL 60637, U.S.A \\ ${ }^{e}$ Kavli Institute for Cosmological Physics, University of Chicago, \\ 5640 South Ellis Avenue, Chicago, IL 60637, U.S.A. \\ E-mail: wenhan@uchicago.edu, zliuphys@umn.edu, mattlow@fnal.gov, \\ liantaow@uchicago.edu
}

ABSTRACT: The measurement of the arrival time of a particle, such as a lepton, a photon, or a pion, reaching the detector provides valuable information. A similar measurement for a hadronic final state, however, is much more challenging as one has to extract the relevant information from a collection of particles. In this paper, we explore various possibilities in defining the time of a jet through the measurable arrival times of the jet constituents. We find that a definition of jet time based on a transverse momentum weighted sum of the times of the constituents has the best performance. For prompt jets, the performance depends on the jet trajectory. For delayed jets, the performance depends on the trajectory of the jet, the trajectory of the mother particle, and the location of the displaced vertex. Compared to the next-best-performing jet time definition, the transverse momentum weighted sum has roughly a factor of ten times better jet time resolution. We give a detailed discussion of the relevant effects and characterize the full geometrical dependence of the performance. These results highlight the critical importance of using a proper definition of jet time with its corresponding detector-dependent calibration and the exciting possibility of deepening our understanding of jets in the time domain.

KEYWORDS: Jets

ARXIV EPRINT: 2109.01682 


\section{Contents}

1 Introduction 1

2 Definitions for jet time 2

3 General behavior 4

3.1 Prompt particles 4

$\begin{array}{lll}3.2 & \text { Delayed particles } & 7\end{array}$

4 Numerical results $\quad 14$

$\begin{array}{lll}4.1 & \text { Simulation details } & 14\end{array}$

$\begin{array}{lll}4.2 & \text { Prompt jets } & 14\end{array}$

$\begin{array}{lll}4.3 & \text { Delayed jets } & 16\end{array}$

5 Conclusions $\quad 19$

$\begin{array}{ll}\text { A Finite length detectors } & 20\end{array}$

$\begin{array}{ll}\text { B Pileup and grooming } & 20\end{array}$

$\begin{array}{lll}\text { C Detector effects } & 22\end{array}$

$\begin{array}{ll}\text { D Hadronization } & 24\end{array}$

$\begin{array}{ll}\text { E Parameter scans } & 24\end{array}$

\section{Introduction}

The time at which a particle arrives at a particular detector layer is a piece of independently measurable and valuable information. Measuring the time of a lepton, a photon, or a hadron has been used extensively at the Large Hadron Collider (LHC) to great effect. ${ }^{1}$ Recently, it was shown that timing information is vital in the search for long-lived particles (LLPs) [7]. The upgraded electronics at the high-luminosity LHC will significantly improve the timing resolution for various subdetectors, reaching tens of picoseconds in some cases, extending the sensitivity of LLP searches even further. For instance, particle timing can improve prompt detection of beyond the Standard Model (BSM) physics [8], enable LLP mass and lifetime determination [9-12], and enhance other various BSM searches [13-38].

Obtaining similar information for final-state quarks and gluons, however, is much more challenging. These particles undergo showering and hadronization before arriving at the

\footnotetext{
${ }^{1}$ Timing has been used in existing searches for heavy stable charged particles [1, 2], stopped particles [3, 4], and non-pointing photons [5, 6], where spatial information is unavailable or ineffective.
} 
detector as a collection of particles, with a corresponding collection of arrival times. A jet is the standard object that combines these particles into a single object that can be used in analyses and searches. In momentum-space variables, summing the four-vectors of the constituents provides a natural definition of the four-vector of the jet. Unfortunately, there is not an obvious choice for the definition of the arrival time of a jet.

The selection of a proper jet time definition is pivotal. A proper definition will enable efficient separation of the Standard Model (SM) prompt background and BSM long-lived signatures. A poor definition, on the other hand, will not allow us to take full advantage of the precision timing capabilities at the level of $30-40$ picoseconds, that will be part of upgrades to ATLAS [39], CMS [40], and LHCb [41]. Already, CMS has demonstrated sensitivity to delayed jets in their search for displaced gluinos [42].

Beyond just performance, a proper definition of jet time may help identify exciting properties of quantum chromodynamics (QCD), enable new jet tagging possibilities, and provide additional inputs for machine learning applications. Even pileup suppression may benefit substantially from an effective usage of jet time because generically pileup vertices have a spread both in space and in time. At the high-luminosity LHC any improvements to pileup suppression are indispensable.

The purpose of this paper is to explore a variety of definitions of jet time and characterize their performances. As with any measurement tool, there are two aspects: accuracy and precision. For jet time, as we will discuss in detail later, the "correct" time is somewhat ambiguous. The precision, or resolution, is well-defined and will be the main criterion in comparing different approaches.

The structure of the paper is as follows. In section 2 we provide a brief overview of various possible definitions of jet time. The general behavior, both for prompt jets and delayed jets, is discussed in section 3. In section 4 we perform an in-depth numerical study of the behavior of each jet time definition, paying special attention to the dependence on the event geometry. Finally, our conclusions are in section 5 along with outlook for future studies. Several appendices are included for cross-checks and studies of additional effects. We discuss the behavior of jet time when endcaps are also used to measure arrival times in appendix A, the impact of pileup and jet grooming in appendix B, the effects of detector resolution in appendix $\mathrm{C}$, and the impact of hadronization in appendix $\mathrm{D}$.

\section{Definitions for jet time}

In this section we briefly describe the definitions of jet time that we study. More detailed descriptions will follow in section 3 and simulation results will be shown in section 4 .

We first define our notation. A single particle $i$ has a four-momentum $\left(E_{i}, \vec{p}_{i}\right)$ and a particle time $t_{i}$, which is the time that it crosses a particular layer of the detector. A jet $J$ is a set of particles which we write as $J=\{i\}$. While a particle has an unambiguous time, the jet has a set of times $\left\{t_{i}\right\}$ associated to it. In the same way that it is often useful to treat the jet as a single four-vector, e.g. in new physics searches, it is also useful to be able to assign a jet a single time $t_{J}$, that we call the jet time. 
There are a number of possibilities that can be used. One can choose a single constituent $i^{\prime}$ in the jet and use its particle time $t_{i^{\prime}}$ to represent the jet time. Jet time definitions of this type include:

- median time: take $t_{J}$ to be the median value of the particle times $\left\{t_{i}\right\}$,

- hardest time: take $t_{J}$ to be the time $t_{i_{h}}$, that corresponds to the time of the constituent $i_{h}$ with the largest transverse momentum,

- random time: take $t_{J}$ to be a randomly-drawn value of the particle times $\left\{t_{i}\right\}$.

The median time has been used by CMS in their search for gluinos with displaced decays [42]. The hardest particle in a jet is likely to be very close to the jet axis so it may be a good proxy for the time of the jet. We do not expect choosing a random particle time as the jet time to perform well, but it is useful as a baseline comparison.

Another option is to calculate $t_{J}$ from a weighted sum of $\left\{t_{i}\right\}$, similar to a jet shape. Generically, this would take the form ${ }^{2}$

$$
t_{J}^{(\alpha, \beta, \gamma)} \propto \sum_{i \in \text { jet }}\left(p_{T, i}\right)^{\alpha}\left(\Delta R_{i}\right)^{\beta} t_{i}^{\gamma},
$$

where $\Delta R_{i}$ is the $\eta \phi$-distance between particle $i$ and the jet axis. The two simplest versions of a weighted sum are the average time where $(\alpha, \beta, \gamma)=(0,0,1)$ and the $p_{T}$-weighted time where $(\alpha, \beta, \gamma)=(1,0,1):^{3}$

- average time: take $t_{J}$ to be

$$
t_{J}^{\text {average }}=\frac{1}{N} \sum_{i=1}^{N} t_{i},
$$

where there are $N$ particles in the jet,

- $p_{T}$-weighted time: take $t_{J}$ to be

$$
t_{J}^{p_{T}}=\frac{1}{H_{T, J}} \sum_{i=1}^{N} p_{T, i} t_{i}, \quad H_{T, J}=\sum_{i=1}^{N} p_{T, i},
$$

where $H_{T, J}$ is a normalization factor.

Finally, one could simply disregard the particles times, treat the jet as a single particle, and calculate its time based on the jet kinematics. There are two variations depending on whether the jet is treated as a massless particle or massive particle:

- null time: treat the jet $J$ as a massless particle and calculate its crossing time using the three-momentum $\vec{p}_{J}$ of the jet (assuming knowledge of the production vertex),

\footnotetext{
${ }^{2} \mathrm{~A}$ number of alternatives are possible, such as particle energy $E$ in place of $p_{T}$ or angle $\theta$ in place of $\Delta R$. In a brief survey, we did not find these to outperform the variables used in eq. (2.1).

${ }^{3}$ We briefly studied a few additional cases such as $p_{T}^{2}$-weighted but did not see an improvement over the $p_{T}$-weighted time or average time. An optimization of $\alpha, \beta$, and $\gamma$ is beyond the scope of this work.
} 
- kinematic time: treat the jet $J$ as a massive particle and calculate its crossing time using the four-momentum $\left(E_{J}, \vec{p}_{J}\right)$ of the jet (assuming knowledge of the production vertex).

Since these definitions do not utilize the information available from timing measurements, they do not correspond to experimentally measurable arrival times. They are useful, however, to determine what constitutes good performance from a jet time definition. These times are constant for fixed parent particle trajectory and jet kinematics. In addition, the null time represents the crossing time if the parton did not undergo showering and hadronization and will serve as a useful reference time. ${ }^{4,5}$

After having chosen a definition for $t_{J}$ we also need to choose a metric to evaluate which definition is the most useful. For a jet time definition, we will compare the relative time difference $\Delta t / t_{\text {ref }}$ defined as

$$
\frac{\Delta t}{t_{\mathrm{ref}}}=\frac{t_{J}-t_{J}^{\mathrm{ref}}}{t_{J}^{\mathrm{ref}}}
$$

to determine a good choice.

Each jet has a different value of $\Delta t / t_{\text {ref }}$ so that a sample of jets will lead to a distribution for the relative time difference. The mean of this distribution corresponds to the accuracy of the jet time definition while the width corresponds to the precision, or resolution, of the definition. Since the choice of $t_{\text {ref }}$ is arbitrary it is not obvious that the mean of the distribution is important (not to mention that constant offsets can be corrected in practice). The width of the distribution, on the other hand, is a robust indicator of a stable time definition. For that reason, the width of the relative time difference distribution will be used as the figure of merit when comparing definitions.

\section{General behavior}

In this section, we study analytically the general behavior of the various jet time definitions. We start with the prompt case where the majority of particles originate from the origin and then we move on to the delayed case where the majority of particles originate from a displaced decay.

\subsection{Prompt particles}

For prompt particles we assume that the particles originate at $t=0$ from the origin of the detector $\vec{x}=\overrightarrow{0} \cdot{ }^{6}$ As a detector model, we will consider an infinite cylinder with radius $r_{T} \cdot{ }^{7}$

\footnotetext{
${ }^{4}$ Note that while for prompt jets the null time is computable in data, for delayed jets the null time requires the location of the displaced vertex so it is not always computable in data.

${ }^{5}$ Of the definitions studied, the $p_{T}$-weighted, null, and kinematic times are IRC safe. The hardest time is only IR safe. The average, median, and random times are all IRC unsafe.

${ }^{6} \mathrm{In}$ reality and in simulation, there are displacements from processes like $B$-hadron decays. These have a negligible impact on our analysis.

${ }^{7}$ The differences when endcaps are included are discussed in appendix A.
} 
The time $t_{i}$ of a particle $i$ with four-momentum $\left(E_{i}, \vec{p}_{i}\right)$ is then given by

$$
t_{i}=\frac{r_{T}}{c} \frac{E_{i}}{p_{T, i}}
$$

where $c$ is the speed of light.

For a massless particle this simplifies to

$$
t_{i}=\frac{r_{T}}{c} \frac{\left|\vec{p}_{i}\right|}{p_{T, i}}=\frac{r_{T}}{c} \cosh \eta_{i}
$$

which is a good approximation for particles in a high-momentum jet.

The jet times coming from a single particle within the jet are similarly calculated. For the median, hardest, and random times, the time of the jet is given by the time of the median-time particle $i_{m}$, the hardest particle $i_{h}$, or a random particle $i_{r}$, respectively, and is

$$
t_{J}^{\{\text {median,hardest,random }\}}=t_{\left\{i_{m}, i_{h}, i_{r}\right\}}=\frac{r_{T}}{c} \cosh \eta_{\left\{i_{m}, i_{h}, i_{r}\right\}} .
$$

With the cylindrical detector, the null and kinematic times of the jet, with four-momentum $\left(E_{J}, \vec{p}_{J}\right)$, can also be calculated. The null time of a jet is

$$
t_{J}^{\text {null }}=\frac{r_{T}}{c} \frac{\left|\vec{p}_{J}\right|}{p_{T, J}}=\frac{r_{T}}{c} \cosh \eta_{J}
$$

while the kinematic time of a jet is

$$
t_{J}^{\text {kinematic }}=\frac{r_{T}}{c} \frac{E_{J}}{p_{T, J}}=t_{J}^{\text {null }} \frac{E_{J}}{\left|\vec{p}_{J}\right|} .
$$

In the limit of small jet mass these definitions differ by $\mathcal{O}\left(m_{J}^{2} / \vec{p}_{J}^{2}\right)$.

The average and $p_{T}$-weighted times follow the definitions in eqs. (2.2) and (2.3).

Prompt relative time difference. For jet times using a single particle (median, hardest, and random times), with a time $t_{i}$, the relative time difference using eqs. (3.3) and (3.4) is

$$
\frac{\Delta t}{t_{\mathrm{ref}}}=\frac{t_{i}-t_{J}^{\text {null }}}{t_{J}^{\text {null }}}=\frac{\cosh \eta_{i}}{\cosh \eta_{J}}-1 .
$$

When the particle $i$ points along the same direction as the jet axis, the relative time difference is always zero. When there is a fixed angular distance $\Delta \eta$ between the particle $i$ and the jet axis, however, the relative time difference changes with $\eta_{J}$. Due to the detector geometry, as the jet becomes more forward, the relative time difference will grow.

The furthest that a particle $i$ can be from the axis of the jet is approximately given by the jet radius $R_{\text {jet }}$. Therefore, for a given $\eta_{J}$ there is a maximum relative time difference given by

$$
\left.\frac{\Delta t}{t_{\mathrm{ref}}}\right|^{\max }=\frac{\cosh \left(\eta_{J} \pm R_{\mathrm{jet}}\right)}{\cosh \eta_{J}}-1
$$

where the + applies for positive $\eta_{J}$ and the - applies for negative $\eta_{J}$. 

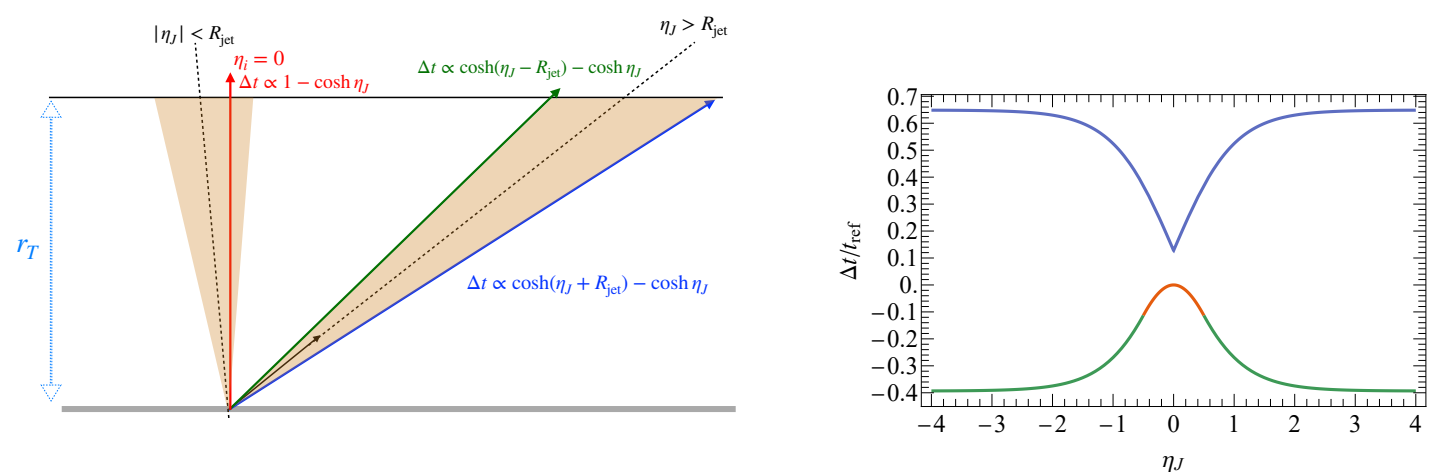

Figure 1. Illustration of the bounds for a prompt jet (left). The slowest particle time, eq. (3.7), follows the high $|\eta|$ boundary of the jet (blue). The fastest particle time, eq. (3.8), follows the low $|\eta|$ boundary of the jet (green) or the $\eta=0$ line (red). Plot of the boundaries for the relative time difference for a jet with $R_{\text {jet }}=0.5$ (right) where the colored lines correspond to the boundaries in the left image.

The minimum is similar except that for $\left|\eta_{J}\right|<R_{\text {jet }}$ there is a stronger bound that comes from the fact that $\eta_{i}=0$ for a massless particle corresponds to the fastest time possible since it is the shortest path from the origin. The bound for $\left|\eta_{J}\right|<R_{\text {jet }}$ consequently only depends on $\eta_{J}$. We find

$$
\left.\frac{\Delta t}{t_{\mathrm{ref}}}\right|^{\min }= \begin{cases}\operatorname{sech} \eta_{J}-1, & \left|\eta_{J}\right|<R_{\mathrm{jet}}, \\ \frac{\cosh \left(\eta_{J} \mp R_{\mathrm{jet}}\right)}{\cosh \eta_{J}}-1, & \text { else. }\end{cases}
$$

Eqs. (3.7) and (3.8) taken together specify boundaries in the space of pseudorapidity vs. relative time difference. The different jet time definitions will have different distributions within these boundaries. Figure 1 illustrates these boundaries graphically.

At $\eta_{J}=0$ the minimum of $\Delta t / t_{\text {ref }}$ is 0 while the maximum is $\cosh \left(R_{\text {jet }}\right)-1>0$ which means that the relative time difference cannot be negative. Therefore, for very central jets we expect the relative time difference distributions to skew towards positive values since there is more available phase space. For less central jets we do not expect a strong preference for positive or negative values based only on phase space.

For the kinematic time rather a bound, one can relate the relative time difference to a kinematic quantity. From eqs. (3.4) and (3.5) we find that for the kinematic time

$$
\frac{\Delta t}{t_{\mathrm{ref}}}=\frac{E_{J}}{\left|\vec{p}_{J}\right|}-1=\frac{1}{\beta_{J}}-1,
$$

where $\beta_{J}$ is the velocity. Since $\beta_{J} \leq 1$ the relative time difference for the kinematic time is always non-negative. This is expected since the kinematic time points in the same direction as the null time, but adjusts for the mass of the jet.

Next, we move to the relative time difference for the $p_{T}$-weighted time. This has a simple form given our cylindrical detector model. The $p_{T}$-weighted time is

$$
t_{J}^{p_{T}}=\frac{1}{H_{T, J}} \sum_{i=1}^{N} p_{T, i} t_{i}=\frac{1}{H_{T, J}} \sum_{i=1}^{N} \frac{r_{T}}{c} E_{i}=\frac{r_{T}}{c} \frac{E_{J}}{H_{T, J}},
$$


and the corresponding relative time difference is

$$
\frac{\Delta t}{t_{\mathrm{ref}}}=\frac{E_{J}}{H_{T, J}} \frac{p_{T, J}}{\left|\vec{p}_{J}\right|}-1=\frac{E_{J}}{\left|\vec{p}_{J}\right|} \frac{p_{T, J}}{H_{T, J}}-1 .
$$

Written in the form after the second equality we recognize the factor $E_{J} /\left|\vec{p}_{J}\right| \geq 1$ from eq. (3.9). The other factor $p_{T, J} / H_{T, J}$ is the ratio of the jet $p_{T}$ to the scalar sum of the constituent $p_{T}$ values. Since $p_{T, J}$ is a vector sum we have $p_{T, J} / H_{T, J} \leq 1$. The distribution at a given $\eta_{J}$ is determined by the $\eta$-dependence of each of these two terms.

For small mass jets $E_{J} /\left|\vec{p}_{J}\right| \approx 1+m_{J}^{2} / \vec{p}_{J}^{2}$ and schematically for QCD jets the mass is $\left\langle m_{J}^{2}\right\rangle \sim R^{2} p_{T, J}^{2} \sim R^{2} \vec{p}_{J}^{2} \operatorname{sech}^{2} \eta_{J}$ [43]. Consequently, $E_{J} /\left|\vec{p}_{J}\right| \sim 1+R^{2} \operatorname{sech}^{2} \eta_{J}$ which peaks at $\eta_{J}=0$ and reduces as $\left|\eta_{J}\right|$ grows. The other quantity $p_{T, J} / H_{T, J}$ depends on the energy distribution in the jet and is not strongly correlated with $\eta_{J}$. Therefore, we expect that the relative time distribution for the $p_{T}$-weighted time to be positively-skewed for central jets and switch over to negatively-skewed as the jets become more forward.

\subsection{Delayed particles}

Next, we study the jet time behavior for delayed particles. Our benchmark scenario involves a mother particle $M$ that travels a macroscopic distance, then decays into two daughter particles $D$ and $\widetilde{D}$. We assume that $M$ and $\widetilde{D}$ are unobserved while $D$ is colored and results in a jet due to showering and hadronization. ${ }^{8}$ In our numerical study we take $M$ as a gluino, $\widetilde{D}$ as a gravitino, and $D$ as a gluon.

Let the mother $M$ have four-momentum $\left(E_{M}, \vec{p}_{M}\right)$ and decay at the displaced vertex $\vec{x}_{M}$ at a time $t_{M}$. The daughter $D$ showers and hadronizes into a delayed jet. A particle $i$ in the delayed jet has a four-momentum $\left(E_{i}, \vec{p}_{i}\right)$ and originates from $\vec{x}_{M}$ at $t_{M}$. Let the vector pointing from $\vec{x}_{M}$ to where $i$ crosses the detector be $\vec{x}_{i}{ }^{9}$ Since $\left|\vec{x}_{i}\right|$ only makes up a fraction of the total traversed distance, the impact of the per-particle time spread is generically lessened for delayed jets. We will refer to this effect as the daughter time fraction.

If particle $i$ is measured, but the displaced vertex is not identified, then $i$ will be assumed to have come from the origin, having traveled along $\vec{x}_{i}{ }^{\prime}=\vec{x}_{M}+\vec{x}_{i}$. This is illustrated in figure 2. We call the kinematics computed using $\vec{x}_{i}{ }^{\prime}$ the observed kinematics and those using $\vec{x}_{i}$ the truth kinematics.

\begin{tabular}{|c|c|c|}
\hline & four-vector & assumed trajectory \\
\hline truth & $\left(E_{i}, \vec{p}_{i}\right)$ & $\vec{x}_{i}$ \\
\hline observed & $\left(E_{i}, \vec{p}_{i}{ }^{\prime}\right)$ & $\vec{x}_{i}{ }^{\prime}$ \\
\hline
\end{tabular}

For transverse displacements $\gtrsim 10 \mathrm{~cm}$ the tracking efficiency is $\lesssim 40 \%$ in CMS and drops off further above $50 \mathrm{~cm}$ [44]. Conservatively, we assume that the displaced vertex is not

\footnotetext{
${ }^{8}$ If $M$ or $\widetilde{D}$ (or both) are colored, they will propagate as color-neutral $R$-hadrons. They will be unobserved if the resulting $R$-hadrons are electrically-neutral.

${ }^{9}$ For simplicity we neglect the effects of curvature in the magnetic field of the detector. For a magnetic field of $3.8 \mathrm{~T}$ the effect on the measurement of time or momentum is less than $1 \%$ for particles with $p_{T}>2.5 \mathrm{GeV}$. In appendix B where we study pileup, we do include curvature induced by the magnetic field.
} 

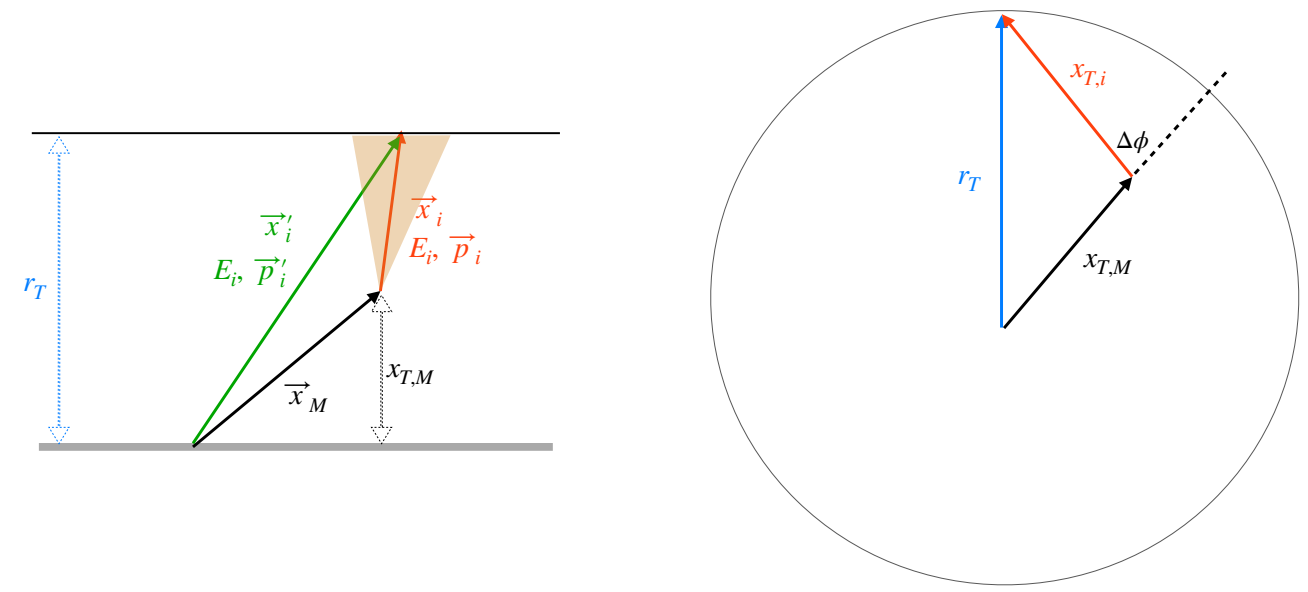

Figure 2. The mother particle $M$ travels along $\vec{x}_{M}$ and decays to a delayed jet (shaded brown). The daughter particle $i$ travels along $\vec{x}_{i}$ until it crosses the detector. If the displaced vertex is not identified then $i$ is assumed to have traveled along $\vec{x}_{i}{ }^{\prime}$.

identified and work with the observed kinematics. The most direct impact of this is that the transverse momentum of any given constituent can be incorrectly assigned. This occasionally leads to noticeable broadening of time definitions that directly depend on the constituents' $p_{T}$. While those that do not, e.g. the median time, are mostly insensitive to whether the truth or observed kinematics are used. A more subtle consequence of using observed kinematics is that the observed opening angle does not correspond to the true opening angle, leading to the inclusion/exclusion of particles with extreme arrival times. We will refer to this discrepancy in opening angles as the effective radius.

In the following, we study in detail these three primary effects which control the performance of the timing of a delayed jet: the daughter time fraction, observed kinematics, and the effective radius.

Observed kinematics. For a massless particle $i$, if it is prompt its time is fully specified by its pseudorapidity $\eta_{i}$ (see eq. (3.2)). When $i$ is delayed, its time depends now on its pseudorapidity $\eta_{i}$, the pseudorapidity of the mother $\eta_{M}$, the azimuthal angle difference $\Delta \phi=$ $\phi_{i}-\phi_{M}$, the speed of the mother $\beta_{M}$, and the transverse decay location of the mother $x_{T, M}$ :

$$
\eta_{M}, \eta_{i}, \Delta \phi, \beta_{M}, x_{T, M} .
$$

The transverse distance $x_{T, i}$ traveled by $i$ is calculated to be

$$
x_{T, i}=\sqrt{r_{T}^{2}-x_{T, M}^{2} \sin ^{2}(\Delta \phi)}-x_{T, M} \cos (\Delta \phi) .
$$

The observed kinematics, $\left(p_{T, i}{ }^{\prime}, \eta_{i}{ }^{\prime}, \phi_{i}{ }^{\prime}\right)$, can be computed in terms of the variables in eq. (3.13) and $x_{T, i}$. The observed pseudorapidity $\eta_{i}{ }^{\prime}$ is found from solving

$$
r_{T} \sinh \left(\eta_{i}{ }^{\prime}\right)=x_{T, M} \sinh \left(\eta_{M}\right)+x_{T, i} \sinh \left(\eta_{i}\right) .
$$

In terms of the true transverse momentum $p_{T, i}$, the observed transverse momentum $p_{T, i}{ }^{\prime}$ is

$$
p_{T, i} \sqrt{1+\left(\frac{x_{T, M}}{r_{T}} \sinh \left(\eta_{M}\right)+\frac{x_{T, i}}{r_{T}} \sinh \left(\eta_{i}\right)\right)^{2}}=p_{T, i} \cosh \left(\eta_{i}\right) .
$$




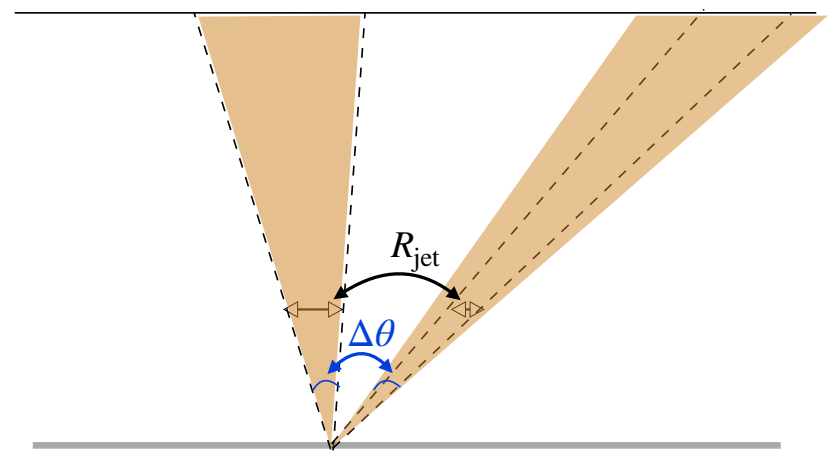

Figure 3. Illustration of $\Delta \theta$ effect for prompt jets. The dashed lines depict jets with the same $R_{\text {jet }}$ while the shaded regions depict jets with the same $\Delta \theta$.

Finally, the observed azimuthal angle $\phi_{i}{ }^{\prime}$ is

$$
\tan \left(\phi_{i}{ }^{\prime}\right)=\frac{x_{T, M} \sin \left(\phi_{M}\right)+x_{T, i} \sin \left(\phi_{i}\right)}{x_{T, M} \cos \left(\phi_{M}\right)+x_{T, i} \cos \left(\phi_{i}\right)}
$$

Jets are clustered using the observed kinematics. The time $t_{i}$ of a particle $i$ is not impacted by using observed kinematics because the arrival time of a particle is an independent measurement. Since $\vec{x}_{i}$ and $\vec{x}_{i}{ }^{\prime}$ cross the detector at the same location, the effect on clustering using observed kinematics is nearly negligible (comparable to the difference between different jet algorithms).

The primary impact of using observed kinematics is on jet time definitions that utilize $p_{T}$ information. We expect the $p_{T}$-weighted time to be impacted at a noticeable level (the size of this effect will be studied in section 4). The hardest time could be affected if which jet constituent is the hardest changes under the observed kinematics. In practice, this is rare due to the hierarchical nature of the parton shower. The median time, likewise, is minimally affected.

Effective radius. The radius of a jet, $R_{\text {jet }}$, is a parameter in the jet finding algorithm that determines which particles are included in the same jet. It determines the catchment area of a jet in $\eta \phi$-space which is approximately a circle with radius $R_{\text {jet }}$ for cone-like algorithms used on isolated jets [45].

When choosing a jet radius there are trade-offs. If the radius is too small, then particles coming from the showering of a hard particle could fall outside a particular jet and the jet will not be a useful proxy for the underlying hard quark or gluon. If the radius is too large, the jet is more susceptible to contamination like underlying event and pileup [43].

One consequence for prompt jets of using a fixed $R_{\text {jet }}$ for jet finding is that an optimal jet radius may be different for central jets as compared to forward jets. This is because for a fixed $R_{\text {jet }}$ in $\eta \phi$-space, the corresponding angular distance, $\Delta \theta$, between a pair of particles is smaller for forward jets than for central jets. Physically, if a set of central particles within a jet with radius $R_{\text {jet }}$ were shifted to larger $|\eta|$ values, then they may not all fit within a radius $R_{\text {jet }}$ anymore.

In the prompt case, we consider the effective radius of the jet to be the angular distance $\Delta \theta$ that is required to keep $\Delta R$ fixed. This means that forward jets have a smaller effective 


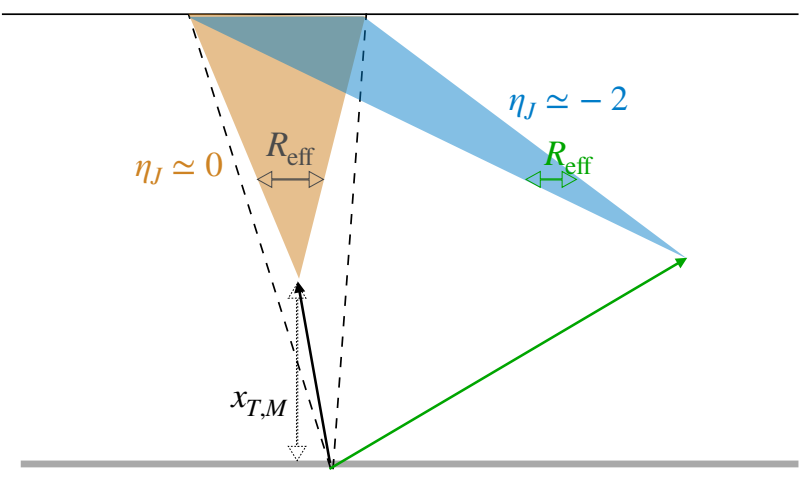

Figure 4. Illustration of $R_{\text {eff }}$ effect for delayed jets. The brown shaded region has a larger $R_{\text {eff }}$ compared to the dashed lines because $x_{T, M}$ is larger. The blue shaded region shows both the effect of shifting and tilting.

radius than central jets because for fixed $\Delta R$ the required $\Delta \theta$ distance shrinks. See figure 3 for an illustration. Variable $R$ jets were proposed to account for this by letting the jet radius grow at larger $|\eta|$ by scaling the radius inversely with transverse momentum [46].

In contrast, for delayed jets we consider the effective radius of a jet to be the true $\Delta R$ distance that is required to keep observed $\Delta R$ fixed. There are two factors that alter the effective radius for delayed jets. The first is that a non-zero value of $x_{T, M}$ means the jet originates closer to the detector radius $r_{T}$. The same way that the image from a projector is smaller as you move the projector closer to the screen, a fixed observed $\Delta R$ value corresponds to a larger true $\Delta R$ value as $x_{T, M}$ grows. See figure 4 for an illustration.

The second effect is that both $\eta_{J}$ and $\eta_{M}$ can vary. Changing $\eta_{J}$ tilts the direction of the jet and generally causes the effective radius to shrink with $\eta_{J}$ similar to the prompt case. Changing $\eta_{M}$ is not a tilt, but rather a shift of the origin point of the particles. Due to the geometry of $\eta \phi$-space the effective radius generally increases as $\left|\eta_{M}\right|$ grows.

The effective radius can be estimated numerically. As shorthand we write the observed pseudorapidity of a jet as $\eta_{J}{ }^{\prime}=f\left(\eta_{M}, x_{T, M}, \eta_{J}\right)$ where the function is found in eq. (3.15). We define the effective jet radius as $R_{\text {eff }}$ and find it by solving

$$
R_{\mathrm{jet}}=f\left(\eta_{M}, x_{T, M}, \eta_{J}+\frac{1}{2} R_{\mathrm{eff}}\right)-f\left(\eta_{M}, x_{T, M}, \eta_{J}-\frac{1}{2} R_{\mathrm{eff}}\right)
$$

The definition is not rigorous but rather is meant to provide intuition for the general behavior. We also set $\Delta \phi$ zero in the above for simplicity. In figure 5 we plot $R_{\text {eff }}$ as a function of $\eta_{J}$ for several sample points of $x_{T, M}$ and $\eta_{M}$ with fixed jet radius $R_{\text {jet }}=0.5$.

For isolated QCD jets, we expect the jet properties to change slowly with respect to increasing $R_{\text {eff. }}$. In a typical parton shower there are both more and higher momentum particles near the center of the jet. Including additional soft particles further from the jet 


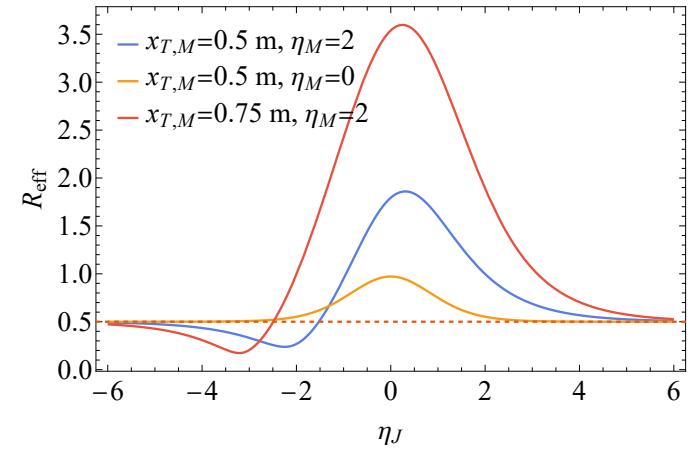

Figure 5. The effective radius, $R_{\text {eff }}$, as a function of $\eta_{J}$ with $x_{T, M}=0.5 \mathrm{~m}$ and $\eta_{M}=2$ (blue solid line), $x_{T, M}=0.5 \mathrm{~m}$ and $\eta_{M}=0$ (yellow solid line), and $x_{T, M}=0.75 \mathrm{~m}$ and $\eta_{M}=2$ (red solid line). A fixed value of 0.5 is also shown (red dashed line).

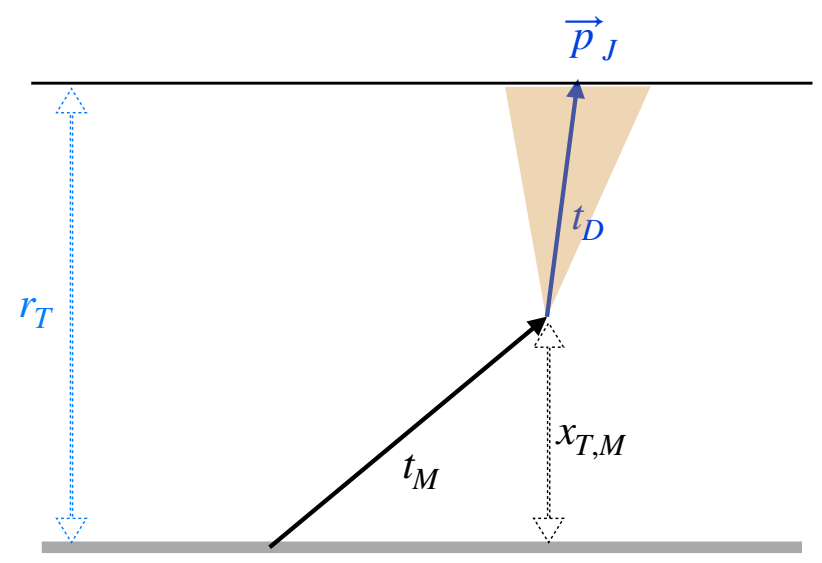

Figure 6. The time of flight of the mother particle is $t_{M}$ and the time of flight of a daughter particle is $t_{D}$.

axis will not perturb the jet four-vector by much. When $R_{\text {eff }}$ decreases the jet properties should change faster as more and higher momentum particles are excluded.

Daughter time fraction. The third effect is the fraction of time that comes from time of flight of the daughter $i$ as compared to the time of flight of the mother, as shown in figure 6. Intuitively, when the mother travels more of the distance from the origin to the detector, there is less variation among the times of the particles in a jet. Consequently, the distribution of jet times becomes narrower.

The time of a delayed particle $i$ is

$$
t_{i}=t_{M}+\frac{\left|\vec{x}_{i}\right|}{c} \frac{E_{i}}{\left|\vec{p}_{i}\right|}=t_{M}+\frac{x_{T, i}}{c} \frac{E_{i}}{p_{T, i}} .
$$

The null time of a jet, which we continue to use as the reference time, is

$$
t_{J}^{\text {null }}=t_{M}+\frac{x_{T, J}}{c} \frac{\left|\vec{p}_{J}\right|}{p_{T, J}}=t_{M}+\frac{x_{T, J}}{c} \cosh \eta_{J} .
$$




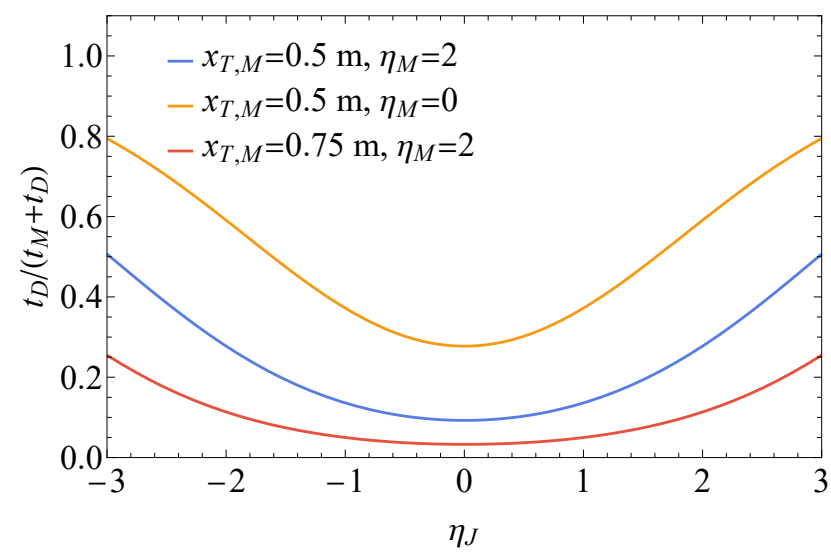

Figure 7. The overall suppression in the width due to the daughter time fraction as a function of $\eta_{J}$ using $\beta_{M}=0.4$ with $x_{T, M}=0.5 \mathrm{~m}$ and $\eta_{M}=2$ (blue), $x_{T, M}=0.5 \mathrm{~m}$ and $\eta_{M}=0$ (yellow), and $x_{T, M}=0.75 \mathrm{~m}$ and $\eta_{M}=2$ (red).

Let us first consider the relative time difference for the median time so that $i=i_{m}$. We have

$$
\frac{\Delta t}{t_{\mathrm{ref}}}=\frac{t_{i}-t_{J}^{\text {null }}}{t_{J}^{\text {null }}}=\frac{\left(t_{M}+\frac{x_{T, i}}{c} \frac{E_{i}}{p_{T, i}}\right)-\left(t_{M}+\frac{x_{T, J}}{c} \frac{\left|\vec{p}_{J}\right|}{p_{T, J}}\right)}{t_{M}+\frac{x_{T, J}}{c} \frac{\left|\vec{p}_{J}\right|}{p_{T, J}}} .
$$

We set $t_{D} \equiv\left(x_{T, J} / c\right)\left(\left|\vec{p}_{J}\right| / p_{T, J}\right)$, representing the "null" time from the daughter segment. If we approximate $x_{T, i}=x_{T, J}$ (i.e. the jet is narrow) and particle $i$ as massless, then we find

$$
\frac{\Delta t}{t_{\mathrm{ref}}}=\frac{t_{D}}{t_{M}+t_{D}}\left(\frac{\cosh \eta_{i}}{\cosh \eta_{J}}-1\right)
$$

The first factor is the fraction of the particle's time that is traveled by the daughter and the second factor we recognize from eq. (3.6) as the prompt distribution evaluated at particle $i$ 's true pseudorapidity. As the distance the daughter travels, $x_{T, J}$, shrinks, so does the spread in $\Delta t / t_{\text {ref }}$. In figure 7 , we plot the suppression factor, $t_{D} /\left(t_{M}+t_{D}\right)$, as a function of $\eta_{J}$ for several sample points of $x_{T, M}$ and $\eta_{M}$ using $\beta_{M}=0.4$

Delayed relative time difference. Here we briefly review our expectations for the relative time difference in delayed jets. Recall that for single particle measures, like the median or the hardest, the prompt relative time is given by

$$
\frac{\Delta t}{t_{\mathrm{ref}}}=\frac{\cosh \eta_{i}}{\cosh \eta_{J}}-1
$$

The three effects that cause the delayed distribution to differ from eq. (3.23) are:

- the daughter time fraction,

- the effective radius of the jet,

- the difference between observed and truth kinematics. 
Let us now contrast a few jet time definitions to assess the impact of each delayed effect on the relative time distribution. We start with the hardest time. The difference in observed kinematics should have a negligible effect except in rare instances when the hardest particle in a jet changes between observed and truth kinematics. The effective radius should also have a minimal effect because the hardest particle in a jet tends to be near the jet axis. The daughter time fraction, however, is an irreducible effect.

From eq. (3.22) we see that the delayed distribution inherits the prompt dependence on the daughter's true pseudorapidity, but with an additional suppression from the fact that spread between particles occurs over a smaller distance. The suppression comes from the prefactor

$$
\frac{t_{D}\left(x_{T, M}, \eta_{i}\right)}{t_{D}\left(x_{T, M}, \eta_{i}\right)+t_{M}\left(x_{T, M}, \eta_{M}, \beta_{M}\right)} .
$$

The times scale with their respective pseudorapidities, $t_{M} \propto \cosh \left(\eta_{M}\right)$ and $t_{D} \propto \cosh \left(\eta_{i}\right)$, so that the prefactor is closest to 1 when $\eta_{D} \approx 0$ and $\left|\eta_{i}\right|$ is large, and closest to 0 when $\eta_{i} \approx 0$ and $\left|\eta_{D}\right|$ is large. The prefactor can range from 0 to 1 and it plays a large role in the relative time difference distribution.

Next, we consider the median time. Again, we expect the observed kinematics to have a negligible effect on the relative time difference. The effective radius, however, can now have an impact because each particle has an equal effect on the median time of a jet. As $R_{\text {eff }}$ grows, particles further from the jet axis are included in the jet and in the calculation of the jet time. Being far from the jet axis, these particles act like noise for the particle time distribution leading to more variation in the relative time difference distribution. Conversely, a shrinking $R_{\text {eff }}$ will tend to narrow the distribution somewhat. The daughter time fraction is irreducible and has an $\mathcal{O}(1)$ effect on the median time.

Finally, we consider the $p_{T}$-weighted time. The daughter time fraction is again a driving effect. The impact of the effective radius should be smaller than in the median case, because particles far from the jet axis are typically soft so their contribution to the $p_{T}$-weighted time is suppressed by their $p_{T}$. The observed kinematics, however, can now have a large effect. The $p_{T}$-weighted time for delayed jets is

$$
t_{J}^{p_{T}}=\frac{1}{H_{T, J^{\prime}}} \sum_{i=1}^{N} p_{T, i}{ }^{\prime} t_{i}, \quad H_{T, J^{\prime}}{ }^{\prime}=\sum_{i=1}^{N} p_{T, i}{ }^{\prime} .
$$

From eq. (3.16) we see that the ratio $p_{T, i}{ }^{\prime} / p_{T, i}$ is independent of momentum. This means in the infinitely-narrow jet limit the $p_{T}$-weighted time is not affected by the observed kinematics. Beyond this limit, the effect of using the observed $p_{T}$ can be large if the variation of $p_{T, i}{ }^{\prime} / p_{T, i}$ is large over the area of the jet.

The ability to accurately identify displaced vertices can eliminate the impact of the observed kinematics. Such an upgrade would be expected to improve the performance of the $p_{T}$-weighted time, but have a small effect on the hardest time and the median time. 


\section{Numerical results}

In this section, we compute the relative time differences for several jet time definitions in simulated data. Results will be compared with the derived behaviors from section 3 and are found to follow the predicted trends.

\subsection{Simulation details}

For prompt jets, we generate $p p \rightarrow Z^{\prime} \rightarrow q \bar{q}$ events, where $q=u, d$, using Pythia v8.240 [47] at a center of mass energy of $\sqrt{s}=14 \mathrm{TeV}$ and with a $Z^{\prime}$ mass of $m_{Z^{\prime}}=1 \mathrm{TeV}$. Initial state radiation (ISR) and multiparton interactions (MPI) are turned off. Particles with $p_{T}<0.5 \mathrm{GeV}$ or with $|\eta|>4$ are discarded. Particles are clustered into anti- $k_{T}$ jets [48] with $R_{\text {jet }}=0.5$ using FastJet v3.3.2 [49]. The results are presented at particle-level without any detector resolution or time resolution included. The impact of these effects is shown in appendix $\mathrm{C}$ to be small.

For delayed jets, we generate $p p \rightarrow \tilde{g} \tilde{g} \rightarrow(g \tilde{G})(g \tilde{G})$ at parton-level using MadGraph5 v2.7.3 [50] at a center of mass energy of $\sqrt{s}=14 \mathrm{TeV}$ and with particle masses of $m_{\tilde{g}}=$ $2 \mathrm{TeV}$ and $m_{\tilde{G}}=10^{-16} \mathrm{TeV} .{ }^{10}$ Events are showered with Pythia with ISR and MPI turned off. Particles with an observed transverse momentum below $p_{T}{ }^{\prime}<0.5 \mathrm{GeV}$ or with an observed pseudorapidity $\left|\eta^{\prime}\right|>4$ are discarded, where a cylindrical detector with a radius of $r_{T}=1 \mathrm{~m}$ is used.

In both the prompt and delayed samples, hadronization is turned off. In Pythia, when there are both prompt and displaced particles, due to the hadronization procedure, some particles that descend from the displaced gluon can be assigned to the prompt vertex. Using unhadronized events avoids the issue of determining to which vertex a hadron should be assigned. In appendix D we compare the relative time distributions, in a prompt sample, with and without hadronization and find that the impact is at most a few percent.

\subsection{Prompt jets}

We first look at prompt jets because the prompt distributions are inputs to understanding the delayed distributions. In each event we only consider the hardest jet and require that it has $p_{T}>250 \mathrm{GeV}$.

In figure 8 (left) we show the distribution of $\Delta t / t_{\text {ref }}$ for jets with $\left|\eta_{J}\right|<0.5$ for the jet time definitions of $p_{T}$-weighted, median, hardest, average, and random. As expected, selecting a random particle in the jet to represent the jet time yields the widest distribution. Its distribution skews towards positive relative times because $\eta_{J}=0$ corresponds to the fastest possible time, meaning there is more phase space for positive values. The other time definitions have narrower distributions but still skew towards positive values. The median, hardest, and average times have comparable performance, while the $p_{T}$-weighted time has the narrowest distribution.

From figure 8 we see that each $\Delta t / t_{\text {ref }}$ distribution peaks near zero, but that the mean depends on the range of $\eta_{J}$ used. The width of the distributions is an indicator

\footnotetext{
${ }^{10}$ Whether using gluon-initiated or light quark-initiated jets does not give rise to qualitative differences.
} 

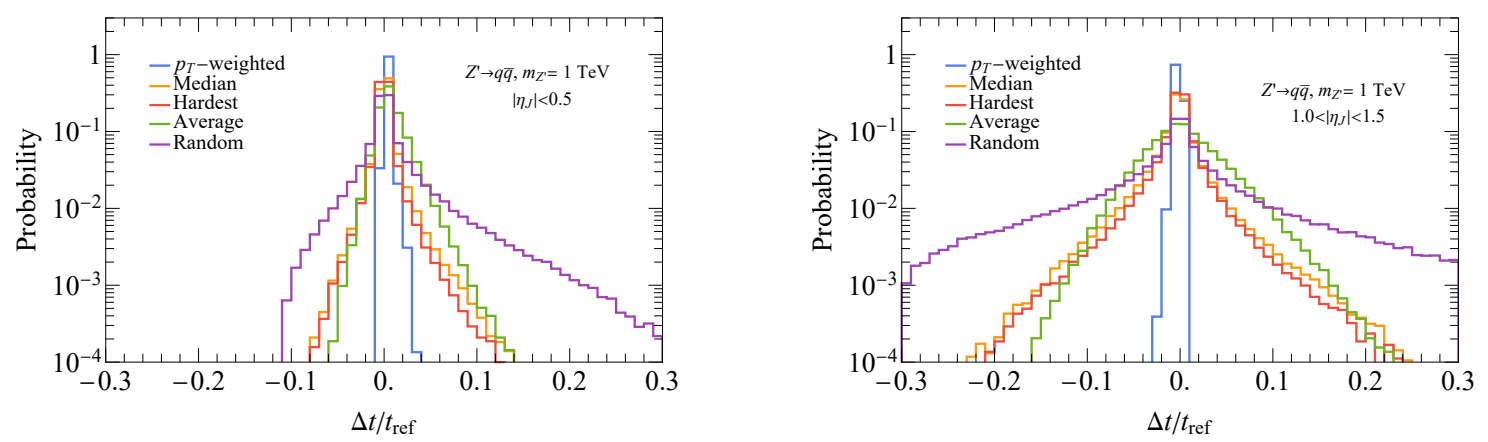

Figure 8. The relative time difference distribution in prompt jets for $\left|\eta_{J}\right|<0.5$ (left) and for $1.0<\left|\eta_{J}\right|<1.5$ (right).

of the resolution of a method and a useful figure of merit. Since these distributions are non-Gaussian, the $1 \sigma$ standard deviation does not fully characterize the shapes, and in particular does not provide useful information about the tails. For that reason, we use the $3 \sigma$ width (i.e. the bounds of the integral containing $99.7 \%$ of events) for comparison. ${ }^{11}$ With this as the resolution, the $p_{T}$-weighted time performs 5 times better than the hardest time and 6 times better than the median time.

In figure 8 (right) we look at the relative time distribution for jets with $1.0<\left|\eta_{J}\right|<1.5$. The same pattern is present here where the random time is the widest distribution, followed by the median, hardest, and average times with similar widths, and the $p_{T}$-weighted time with the narrowest distribution. Again, comparing the resolutions, we find that the $p_{T^{-}}$ weighted time is 16 times better than the hardest time and 17 times better than the median time. The distributions of the median and hardest times widen noticeably in this $1.0<\left|\eta_{J}\right|<1.5$ range, as compared to $\left|\eta_{J}\right|<0.5$, as predicted by eqs. (3.7) and (3.8). The $p_{T}$-weighted time instead depends on the interplay between $E_{J} /\left|\vec{p}_{J}\right|$ and the ratio of transverse momentum to the scalar sum of the constituents' transverse momenta.

To better understand the difference between $\eta_{J}$ regions, we look at the two-dimensional distribution of $\Delta t / t_{\text {ref }}$ vs. $\eta_{J}$. Figure 9 shows this distribution for random time with the bounds from eqs. (3.7) and (3.8) overlaid. The majority of events fill out the region between the bounds with a few events above the maximum, due to mass effects, and a few events below the minimum due to the fact that particles can be slightly farther than $R_{\text {jet }}$ from the jet axis.

Figure 9 (right) shows $\Delta t / t_{\text {ref }}$ vs. $\eta_{J}$ for the median time. The red solid lines are the boundaries from eqs. (3.7) and (3.8). This distribution clusters closer around $\Delta t / t_{\text {ref }}$ values near zero. In fact, the dashed lines are boundaries for a jet with radius $(2 / 3) R_{\text {jet }}$ which corresponds to the empirical observation that the behavior of the median time is similar to choosing a random particle from a narrower jet.

In figure 10 we show the same distribution for the $p_{T}$-weighted time. Here, we see that the behavior predicted by eq. (3.11) does appear in the simulation. The positive relative

\footnotetext{
${ }^{11}$ In fact, we use the minimum width that contains $99.7 \%$ of the events rather than width centered at the mean because of the asymmetric nature of the distributions.
} 

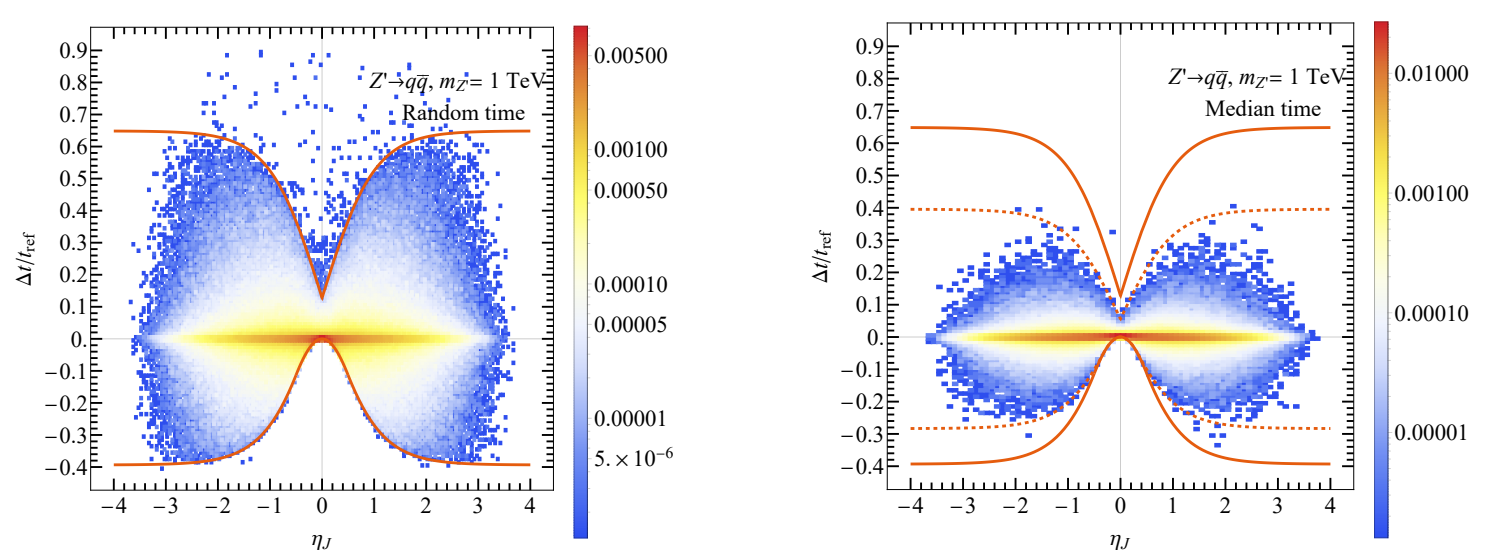

Figure 9. The relative time difference vs. $\eta_{J}$ distribution for random time (left) and median time (right). The solid red curves depict the bounds from eqs. (3.7) and (3.8). The dashed red curve depicts the same bounds for a jet of radius $(2 / 3) R_{\text {jet }}$.

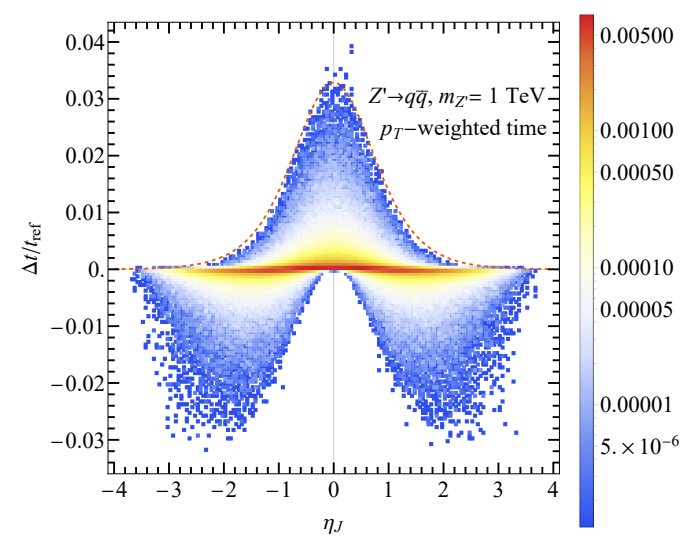

Figure 10. The relative time difference vs. $\eta_{J}$ distribution for $p_{T}$-weighted time. The dashed red curve depicts the relation in eq. (3.11).

time differences near $\eta_{J}=0$ result from the $E_{J} /\left|\vec{p}_{J}\right|$ factor. The shape in that region even follows $\operatorname{sech}^{2}\left(\eta_{J}\right)$ as discussed in section 3.1. As $\left|\eta_{J}\right|$ grows past $\approx 2$ the $E_{J} /\left|\vec{p}_{J}\right|$ factor approaches unity and the $p_{T, J} / H_{T, J}$ factor determines the shape. Both of these factors have a narrow distribution leading to an overall narrow distribution for the relative time difference for the $p_{T}$-weighted time.

\subsection{Delayed jets}

For delayed jets the parameter space expands from $\eta_{J}$ to $\eta_{M}, \eta_{J}, \Delta \phi, \beta_{M}$, and $x_{T, M}$. To study a delayed sample, we vary the values for $\eta_{M}, \eta_{J}$, and $x_{T, M}$ and fix $\Delta \phi=0$ and $\beta_{M}=$ 0.4. The effect of non-zero $\Delta \phi$ has been discussed in our analytic estimates in section 3.2.

In every event, there are two gluinos, each of which decay to a gluon leading to a hard jet. The time of flights in the LHE file was modified such that one of these gluinos is forced to decay outside of the detector while the other gluino is set to have velocity $\beta_{M}$ 

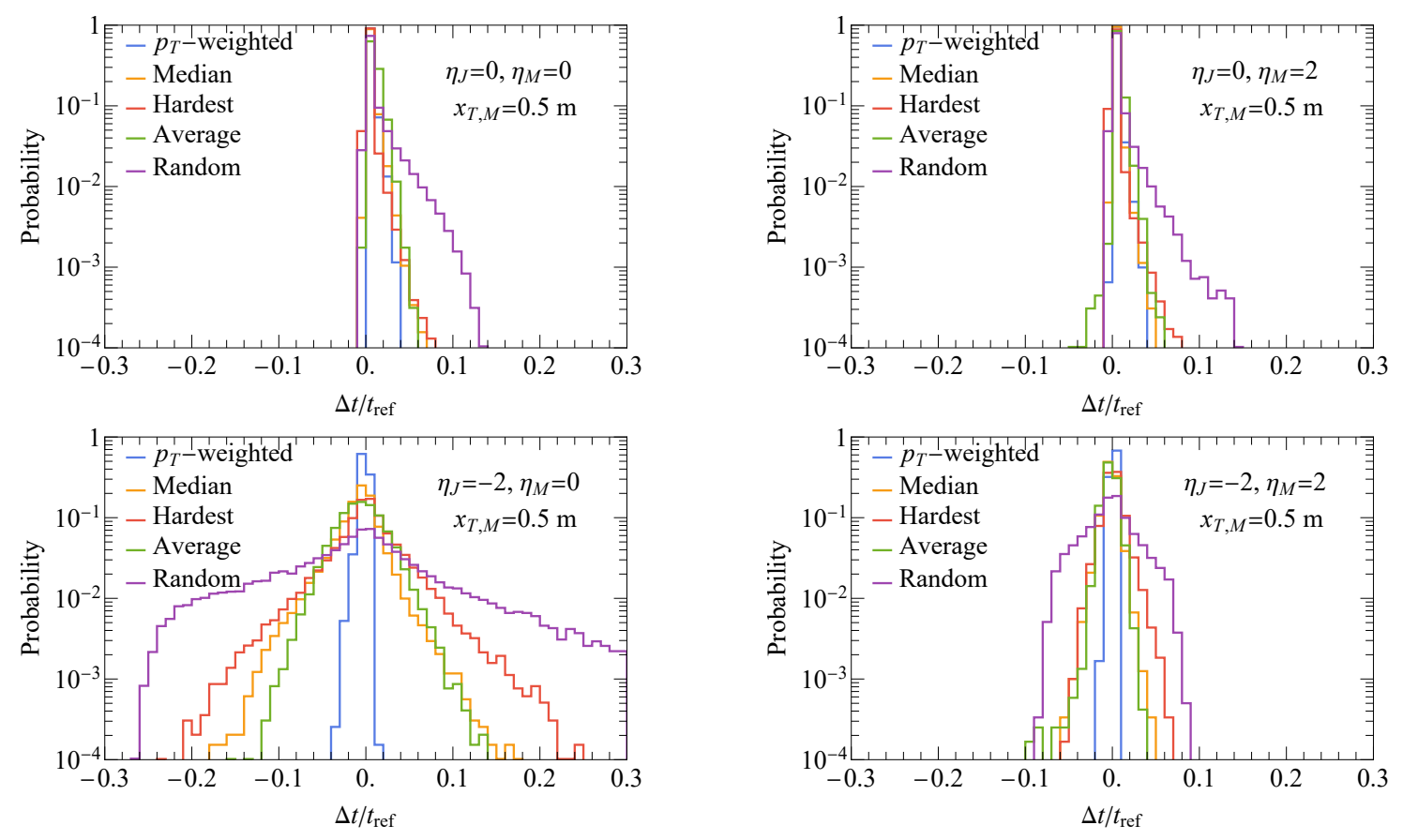

Figure 11. The relative time difference distribution for $\left(\eta_{J}, \eta_{M}\right)=(0,0)$ (top left), $\left(\eta_{J}, \eta_{M}\right)=$ $(0,2)$ (top right), $\left(\eta_{J}, \eta_{M}\right)=(-2,0)$ (bottom left), and $\left(\eta_{J}, \eta_{M}\right)=(-2,2)$ (bottom right) for $x_{T, M}=0.5 \mathrm{~m}$.

and decays at a transverse distance $x_{T, M}$ to a gluon that points along $\eta_{J}$ at parton-level. This same event is then re-showered 100,000 times to produce a sample of jets with fixed parton-level kinematics.

We consider only the hardest jet (that originates from inside the detector) in the event and require it to have an observed $p_{T}{ }^{\prime}>50 \mathrm{GeV}$. In order to identify effects that are dependent on the event topology, we discard events that differ by more than 0.25 in pseudorapidity or 0.25 in azimuthal angle before and after showering.

We first look at distributions of $\Delta t / t_{\text {ref }}$ in figure 11 for $x_{T, M}=0.5 \mathrm{~m}$. The top left plot shows the $p_{T}$-weighted, median, hardest, average, and random times with $\left(\eta_{J}, \eta_{M}\right)=(0,0)$. Much like the prompt case, every definition skews positive since $\eta_{J}=0$ corresponds to fastest possible arrival time. The distributions are narrower than the prompt case due to the decrease in variation in arrival time as captured by the daughter time fraction in eq. (3.24).

Figure 11 (top right) shows the $\Delta t / t_{\text {ref }}$ distributions for $\left(\eta_{J}, \eta_{M}\right)=(0,2)$ which corresponds to a forward gluino that decays to gluon that travels perpendicular to the beamline, directly to the detector. From eq. (3.22) we expect this distribution to be similar to the prompt distribution for central jets. Compared to the $\left(\eta_{J}, \eta_{M}\right)=(0,0)$, this point has a smaller daughter time fraction and is narrower as expected.

The bottom left of figure 11 shows $\Delta t / t_{\text {ref }}$ distributions for $\left(\eta_{J}, \eta_{M}\right)=(-2,0)$. Here the gluino travels perpendicular to the beamline then decays to a backward pointing gluon. The observed pseudorapidity for the gluon is $\eta_{J}{ }^{\prime}=-1.3$. Focusing first on the $p_{T}$-weighted time, we see that the distribution is slightly wider than the prompt case, figure 10, despite a 

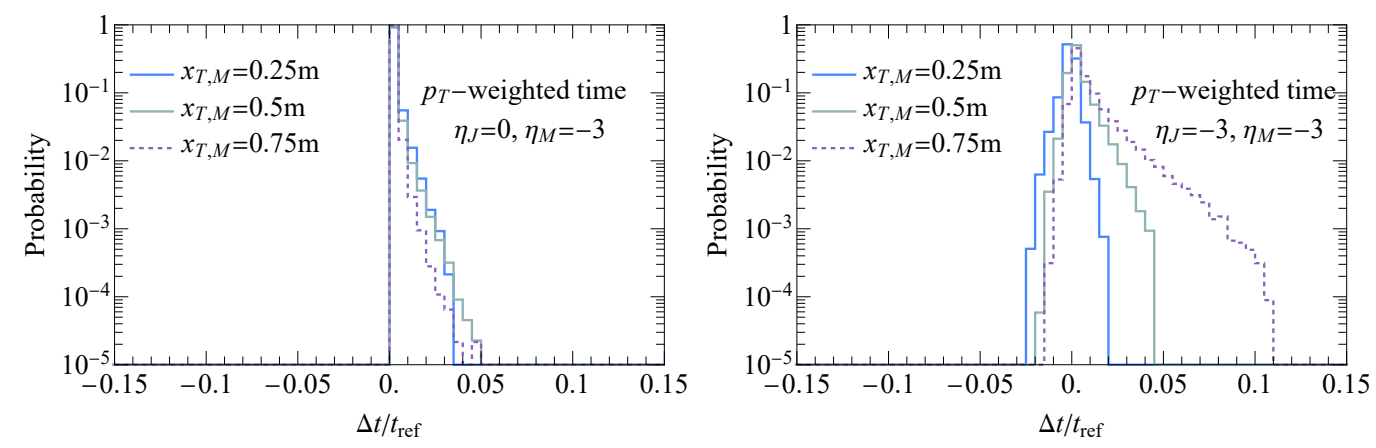

Figure 12. The relative time difference distribution for the $p_{T}$-weighted time at $x_{T, M}=0.25 \mathrm{~m}$ (blue solid line), $0.5 \mathrm{~m}$ (gray solid line), and $0.75 \mathrm{~m}$ (purple dashed line) for $\left(\eta_{J}, \eta_{M}\right)=(0,-3)$ (left) and $\left(\eta_{J}, \eta_{M}\right)=(-3,-3)$ (right).

slight suppression of $\approx 0.6$ from the daughter time fraction. This is due to a sizable variation between the observed and truth kinematics. The median and hardest distributions do not differ much from their prompt counterparts.

Figure 11 (bottom right) shows the distributions for $\left(\eta_{J}, \eta_{M}\right)=(-2,2)$. In this case, each distribution is very narrow. This is primarily a consequence of the jet having $R_{\text {eff }}=$ 0.27. ${ }^{12}$ While the $p_{T}$-weighted distribution is still narrow, the difference is not as large as for other jet times because of the discrepancy between observed and truth kinematics for this configuration.

In figure 12, we plot the distributions of the relative time difference for the $p_{T^{-}}$ weighted time for $x_{T, M}=0.25,0.5,0.75 \mathrm{~m}$. The left plot shows the distributions for $\left(\eta_{J}, \eta_{M}\right)=(0,-3)$ while the right plot shows the distributions for $\left(\eta_{J}, \eta_{M}\right)=(-3,-3)$. In these two plots, we see very different behavior as $x_{T, M}$ increases. The left plot shows the distribution narrowing with $x_{T, M}$, the expected behavior from the decrease in the daughter time fraction. By contrast, the right plot shows the distribution broadening and skewing positive with $x_{T, M}$. This broadening is the expected behavior from both the larger effective cone size, and larger difference between the true and observed $p_{T}$. Furthermore, the relative increase in observed $p_{T}$ is larger forward particles, resulting in the positive skew.

The parameter scans in figures 11 and 12 are useful for emphasizing a few physics points. Firstly, the $p_{T}$-weighted time is consistently better than other jet time definitions across the variation of key kinematics, namely $\eta_{M}$ and $\eta_{J}$. Secondly, the $p_{T}$-weighted time has a different spread at different kinematical points which means one should compute calibrations and efficiencies at each point rather than using a single value over all of parameter space. $^{13}$

\footnotetext{
${ }^{12}$ Note that because the particles in a jet are not uniformly distributed, excluding particles that are farther from the jet axis does result in a narrower relative time difference distribution.

${ }^{13} \mathrm{~A}$ parameter scan of $\left(\eta_{J}, \eta_{M}\right)$ with finer steps can be found in appendix E. The trends shown there agree with the analysis provided here.
} 


\section{Conclusions}

The time of a jet is a theoretically ambiguous and yet experimentally highly relevant quantity. The time profile of a jet provides a new independent probe of jet properties, potentially deepening our understanding of QCD. Experimentally, the jet time is an observable with strong discrimination power in searches for long-lived particles. Like how the jet clustering algorithm itself defines a jet using a collection of particles, the choice of jet time definition determines its properties and performance. A useful definition should have predictable behavior, give the closest representation to the parton-level information, and, more importantly, minimize the spread in arrival time.

In this work, we primarily studied five definitions of jet time. The first was the $p_{T^{-}}$ weighted time where the jet time is a $p_{T}$-weighted sum of the jet constituent arrival times. The second was the median time which uses the median constituent time as the jet time. The third was the hardest time where the time of the highest $p_{T}$ constituent is used as the jet time. The fourth was the average time where the jet time is taken as the average of the constituent times. The fifth was the random time where the time of a constituent was randomly chosen to be used as the jet time.

To evaluate the various definitions, we both predicted and computed in simulation the relative time difference of a definition compared to the time it would take a massless parton to travel along the jet's trajectory. The width of the relative time difference distribution tells us how precisely the jet time can be measured. For prompt jets, we showed that the performance depends on the pseudorapidity of the jet. Due to the geometry of the detector barrel, all jet time definitions have wider distributions as the jets become more forward. We found that the $p_{T}$-weighted jet time consistently has the best performance. For instance, for central jets with $1.0<|\eta|<1.5$, the $p_{T}$-weighted time has a 16 -fold improvement over the (next-best-performing) hardest time. For central jets with $|\eta|<0.5$, the $p_{T}$-weighted time has a 5-fold improvement over hardest time.

For delayed jets, the full kinematics of the event affects the performance. Specifically, the direction of the mother particle, the direction of the jet, and the transverse decay location of the mother particle determine the behavior of the jet times. We show that delayed jet timing behavior can be understood through three effects. The first is the daughter time fraction which is the fact that as the displaced vertex gets closer to the detector there is less distance for the constituents to travel and consequently less spread in their times. The second is the effective radius of the jet that is an effect of the displaced vertex. The third is the that observed $p_{T}$ differs from the true $p_{T}$, which occurs when the displaced vertex is not identified. Just as for prompt jets, the $p_{T}$-weighted time has the best performance over the full parameter space. Furthermore, the strong dependence on the event kinematics emphasizes the importance of having an efficiency map that depends on the long-lived particle's direction, its decay location, and the direction of the daughter jet.

This work is the first study that looks at the impact of different definitions of jet time. There are many related directions that can be explored. For instance, finding the jet time definition that is most amenable to direct calculation may help reduce theory uncertainties. More practically, given the trigger computation complexity budget, it would be useful to 
understand the best alternative jet time definition for a low-level delayed jet trigger. On the analysis side, studies could be done on the interplay between jet time and pileup and grooming. Other new physics models with different event topologies would be interesting to study. More detailed signal-specific studies are needed to evaluate the direct impact of using the jet time in new physics searches. We are optimistic that the jet time has the potential to be a standard tool in long-lived particle searches in the near future.

\section{Acknowledgments}

The authors would like to thank Matthew Citron and Nhan Tran for useful discussions. WHC and LTW are supported by the DOE grant DE-SC0009924. ML acknowledges support from the Fermi Research Alliance, LLC, under Contract No. DEAC02-07CH11359 with the U.S. Department of Energy, Office of Science, Office of High Energy Physics. ZL and LTW acknowledge Aspen Center of Physics for hospitality during the final phase of this study, which is supported by National Science Foundation grant PHY-1607611.

\section{A Finite length detectors}

In the main text, we consider a detector with only a barrel capable of timing measurements. If one includes endcaps, then the timing distributions are different for jets with times that the endcaps would measure.

If the pseudorapidity at which the barrel connects to the endcap is $\eta_{\mathrm{EC}}$, then the arrival time of a particle $i$ at the endcap is

$$
t_{i}^{\text {endcap }}=\frac{z_{\mathrm{EC}}}{c} \frac{1}{\tanh \eta_{i}},
$$

where $z_{\mathrm{EC}}=r_{T} \cosh \eta_{\mathrm{EC}}$. If all of the jet's constituents lie solely in the endcap, the trajectory that yields the shortest (largest) arrival time is now the most forward (central) constituent.

For jets with constituents in the intermediate region, the trajectory that yields the largest arrival time is always the trajectory intersecting the barrel-endcap corner. Depending on the jet axis, the shortest arrival time can be a constituent that intersects the barrel or the endcap.

In figure 13 we show the maximum and minimum relative time differences (for a singleparticle measure) for $r_{T}=1 \mathrm{~m}$ and a total barrel length of $L=6 \mathrm{~m}$ which corresponds to $\eta_{\mathrm{EC}}=1.76 .{ }^{14}$ Once all jet constituents lie within the endcap, the allowed spread in relative time difference sharply drops for prompt jets.

\section{B Pileup and grooming}

We simulate pileup by overlaying $n_{\mathrm{PU}}$ soft $\mathrm{QCD}$ vertices onto our hard event. The number of pileup events is Poisson distributed, with $\left\langle n_{\mathrm{PU}}\right\rangle=140$ and a cutoff at 200 vertices. The

\footnotetext{
${ }^{14}$ This yields the approximate inner geometry of the CMS and ATLAS electromagnetic calorimeters [51, 52].
} 


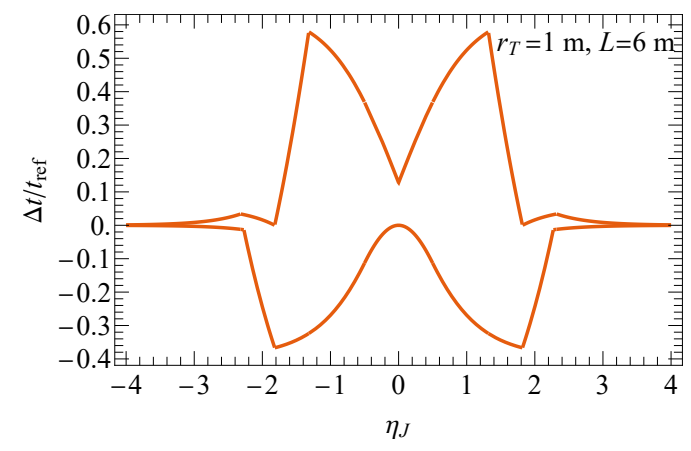

Figure 13. The boundaries for the relative time difference as a function of $\eta_{J}$. This can be compared with the boundaries in figure 9 .
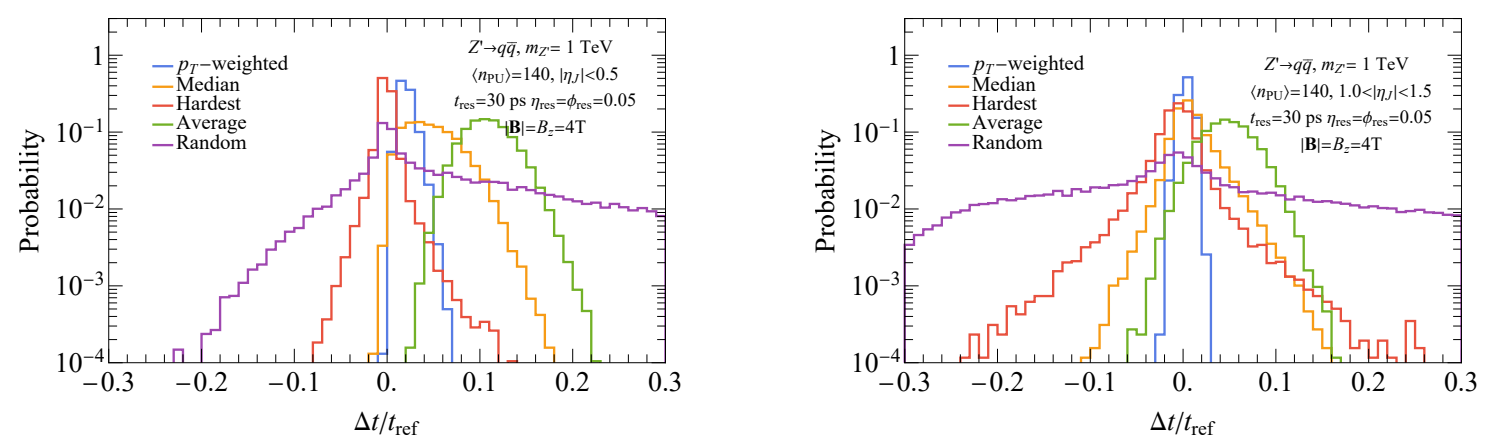

Figure 14. The relative time difference distribution for $\left|\eta_{J}\right|<0.5$ (left) and $1.0<\left|\eta_{J}\right|<1.5$ (right) with pileup and no subtraction. The $\left|\eta_{J}\right|<0.5$ plot can be compared with figure 18 (left) and the $1.0<\left|\eta_{J}\right|<1.5$ plot can be compared with figure 18 (right).

pileup vertices follows a Gaussian spread in both $z$ and $t$, with $\sigma_{z}=c \sigma_{t}=60 \mathrm{~mm}$ [53]. The events with pileup include all of the detector effects discussed in appendix C.

The relative time differences without any form of pileup mitigation are shown in figure 14 for $\left|\eta_{J}\right|<0.5$ (left) and $1.0<\left|\eta_{J}\right|<1.5$ (right). The average time distribution gets distorted for the $\left|\eta_{J}\right|<0.5$ bin, and the peak shifts away from zero considerably. Like those from pileup, low-energy particles have a smaller curvature radius from the magnetic field and are therefore delayed more than higher-energy particles. Since a sizable fraction of a jet's constituents can come from pileup, this causes the average time to shift considerably. The same reasoning is responsible for the broadening of the distributions of the median and $p_{T}$-weighted times. The hardest time is affected very little.

The $1.0<\left|\eta_{J}\right|<1.5$ bin shows less impact from pileup as can be seen, for example, by the peak of the average time distribution remaining close to zero. Similarly, the distributions of the other times broaden slightly, but their peaks do not shift. This is the result of the $p_{T}$ cut restricting to more energetic particles at larger pseudorapidities.

For pileup mitigation, we use an idealized version of charged hadron subtraction [54] where we assume all charged pileup can be removed. The remaining particles were then clustered into $R_{\text {jet }}=0.5$ anti- $k_{T}$ jets and trimmed [55] with $R_{\text {sub }}=0.2$ and $f_{\text {cut }}=0.03$. The choice of keeping $R_{\text {jet }}$ the same is to ensure that the jet times with and without pileup mitigation are directly comparable. 


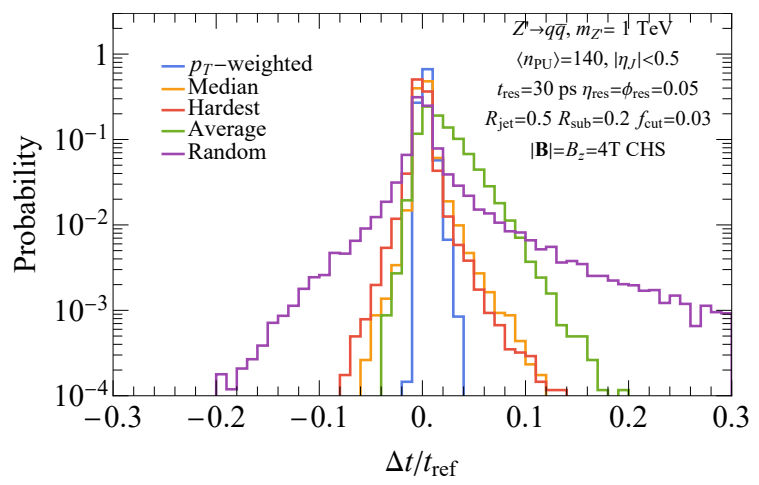

Figure 15. The relative time difference distribution for $\left|\eta_{J}\right|<0.5$ with pileup, charged hadron subtraction, and trimming. This can be compared with figure 14 (left).

The distributions for the $\left|\eta_{J}\right|<0.5$ bin are shown in figure 15 . The improvement is predominantly due to the removal of soft charged pileup particles by charged hadron subtraction. These constituents are the ones that are mainly delayed by mass effects and the magnetic field. Trimming plays a minor role because $R_{\text {sub }}$ is not significantly smaller than $R_{\text {jet }}$ and the number of pileup vertices is large. More aggressive trimming may improve results slightly. ${ }^{15}$

\section{Detector effects}

In this section we show the effects of implementing a simple detector model. We first implement time resolution, followed by time and spatial resolution. The effects are shown in a prompt sample for $\left|\eta_{J}\right|<0.5$ in figure 16 and for $1.5<\left|\eta_{J}\right|<2.0$ in figure 17.

For time resolution, we round each particle's time to the nearest multiple of $30 \mathrm{ps}$. This is the expected resolution of LHC upgrades [39-41]. ${ }^{16}$ The effect on the $\Delta t / t_{\text {ref }}$ distribution can be seen by comparing the left plots (no timing resolution) to the center plots (30 ps timing resolution) in figures 16 and 17 . In both cases, the time resolution has a negligible effect on the shape of the distribution.

For spatial resolution, we consider an $\eta \times \phi$ grid of $0.05 \times 0.05$ cells. The four-momenta are replaced with a massless four-vector with the same energy as the particle, and the direction shifted pointing to the center of the corresponding $\eta \phi$-cell. If multiple particles fall into the same cell and the same time window, their energies are added, and they are combined into a single cell. The effect on the $\Delta t / t_{\text {ref }}$ distribution can be seen in figure 16 (right) and figure 17 (right).

In the $\left|\eta_{J}\right|<0.5$ bin, we see that spatial resolution does not significantly affect the timing distributions. By contrast, the $1.0<\left|\eta_{J}\right|<1.5$ bin has a noticeable broadening in

\footnotetext{
${ }^{15}$ One could also study the performance using pileup mitigation techniques that are better suited to large values of $\left\langle n_{\mathrm{PU}}\right\rangle$ such as jet cleansing [56], constituent subtraction [57], PUPPI [58], soft killer [59], or PUMML [60]. This is beyond the scope of this work.

${ }^{16}$ This time resolution does not include non-Gaussian tail effects (e.g. hot cells) which may affect perparticle arrival time measurements. These effects are very detector-dependent and is beyond the scope of this work.
} 

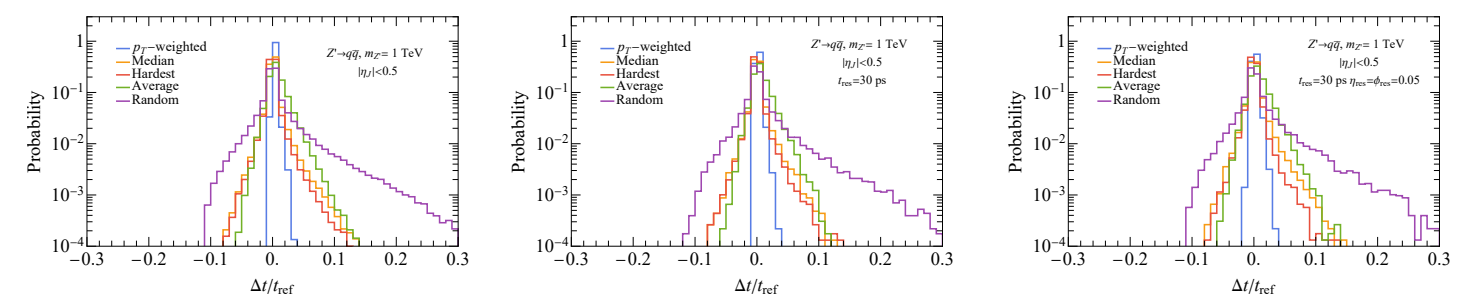

Figure 16. The relative time difference distribution for $\left|\eta_{J}\right|<0.5$ with no detector effects (left), with time resolution added (center), and with time and spatial resolution added (right).
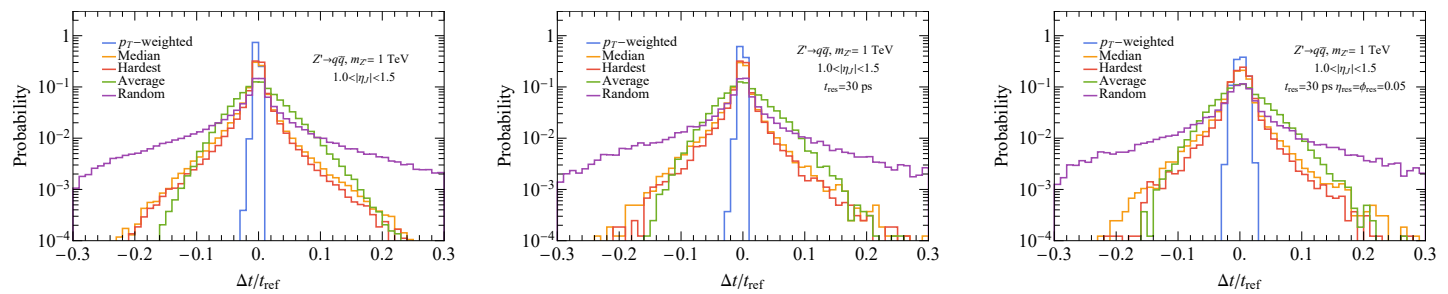

Figure 17. The relative time difference distribution for $1.0<\left|\eta_{J}\right|<1.5$ with no detector effects (left), with time resolution added (center), and with time and spatial resolution added (right).
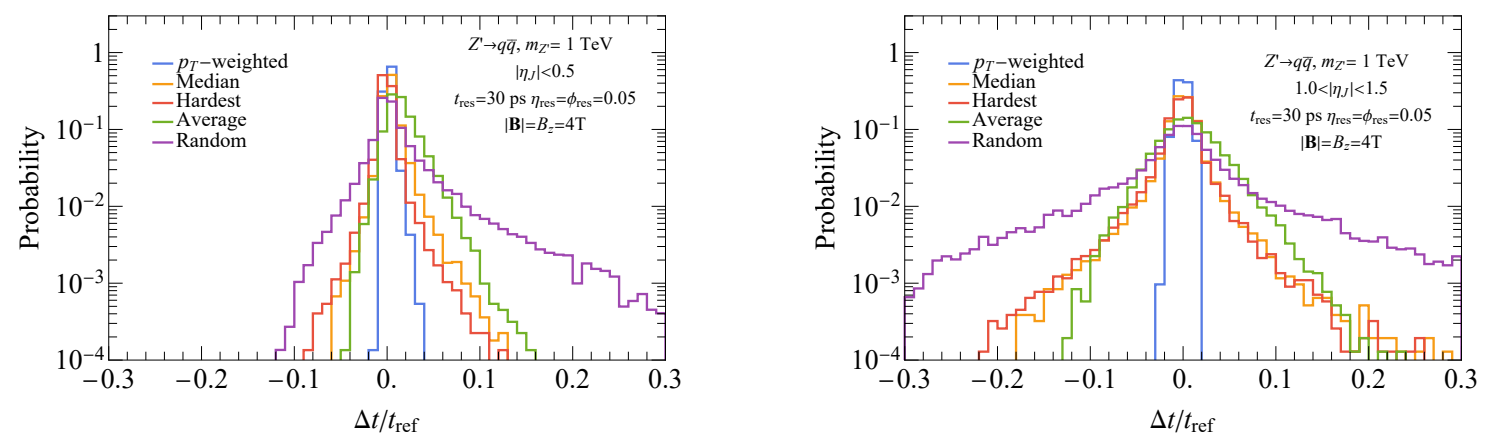

Figure 18. The relative time difference distribution for $\left|\eta_{J}\right|<0.5$ (left) and $1.0<\left|\eta_{J}\right|<1.5$ (right) with a magnetic field. The $\left|\eta_{J}\right|<0.5$ plot can be compared with figure 16 (right) and the $1.0<\left|\eta_{J}\right|<1.5$ plot can be compared with figure 17 (right).

the $p_{T}$-weighted distribution and moderate broadening in the median and hardest distributions. This is because the fractional momentum resolution induced by the spatial resolution increases with $|\eta|$. This impacts both the momentum of the jets and their constituents.

Lastly, we considered the impact of including a $4 \mathrm{~T}$ magnetic field. In this case, the particles were hadronized (in order to get the correct electric charge of the hadrons), and at the same time and spatial resolution was applied. The effect on the relative time distribution for both bins are shown in figure 18. There is a very slight positive pull in the $\left|\eta_{J}\right|<0.5$ bin (left) while the $1.0<\left|\eta_{J}\right|<1.5$ bin (right) has no noticeable change. This difference is due to the $p_{T}>0.5 \mathrm{GeV}$ cut imposed on the constituents. As $\eta$ increases, the energy required to pass the $p_{T}$ cut also increases. The shift in arrival time due to the change in path length is inversely proportional to the energy. 

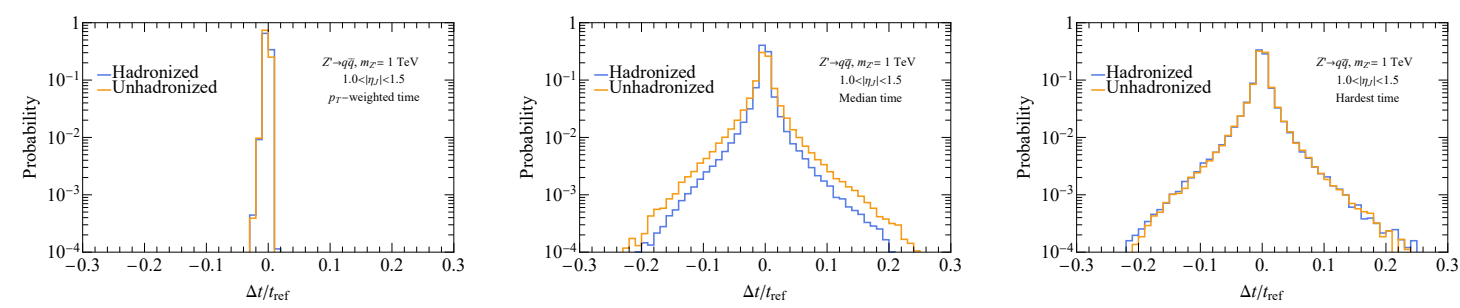

Figure 19. The relative time difference distributions with and without hadronization for the $p_{T^{-}}$ weighted time (left), median time (center), and hardest time (right) with $1.0<\left|\eta_{J}\right|<1.5$.

\section{Hadronization}

In this study the events are not hadronized to ensure that Pythia assigns the correct vertex to each delayed particle. Figure 19 compares the relative time difference for prompt jets with and without hadronization for the $p_{T}$-weighted time (left), median time (center), and hardest time (right) in the range $1.0<\left|\eta_{J}\right|<1.5$. Of the three, only the median time shows a slight observable change.

\section{E Parameter scans}

In figure 20 we show a scan over $\eta_{J}$ in the $x$-direction and $\eta_{M}$ in the $y$-direction for the $p_{T}$-weighted, median, and hardest times. Here, we observe the general trend primarily follows eq. (3.22). Changing $\eta_{J}$ we see that distribution width tends to track with the corresponding pseudorapidity for the prompt distribution. The slight narrowing at large $\left|\eta_{M}\right|$ is due to the changing daughter time fraction, as in eq. (3.24). The few deviations from this pattern are caused by larger changes in the effective radius and additionally from the observed kinematics for the $p_{T}$-weighted time.

Figure 21 shows a scan over $\eta_{J}$ in the $x$-direction and $\eta_{M}$ in the $y$-direction for different values of $x_{T, M}$. Here, we see the same behavior as figure 12. The distribution either narrows or broadens with $x_{T, M}$. The few deviations from this occurs whenever $R_{\text {eff }}$ is very small (e.g. at $\left(\eta_{J}, \eta_{M}\right)=(3,-3)$ for $\left.x_{T, M}=0.5 \mathrm{~m}\right)$. The distributions are narrow over the full parameter space.

Open Access. This article is distributed under the terms of the Creative Commons Attribution License (CC-BY 4.0), which permits any use, distribution and reproduction in any medium, provided the original author(s) and source are credited. 


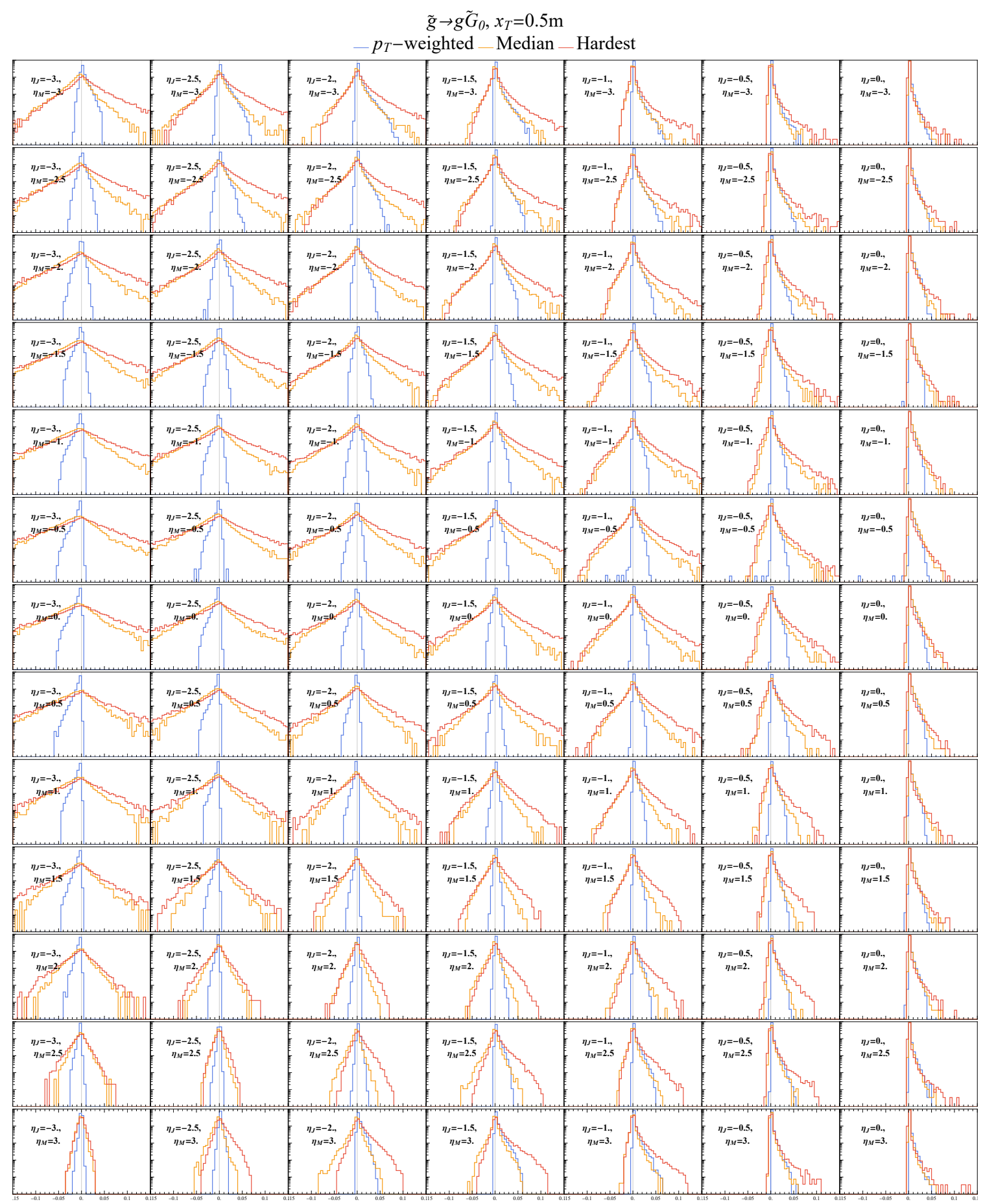

Figure 20. The relative time difference distribution for the $p_{T}$-weighted (blue), median (yellow), and hardest (red) times as a function of $\eta_{J}$ (x-axis) and of $\eta_{M}$ (y-axis) with a transverse decay location of $x_{T, M}=0.5 \mathrm{~m}$. The vertical axis in each plot is in log-scale and ranges from $10^{-5}$ to 1 . 

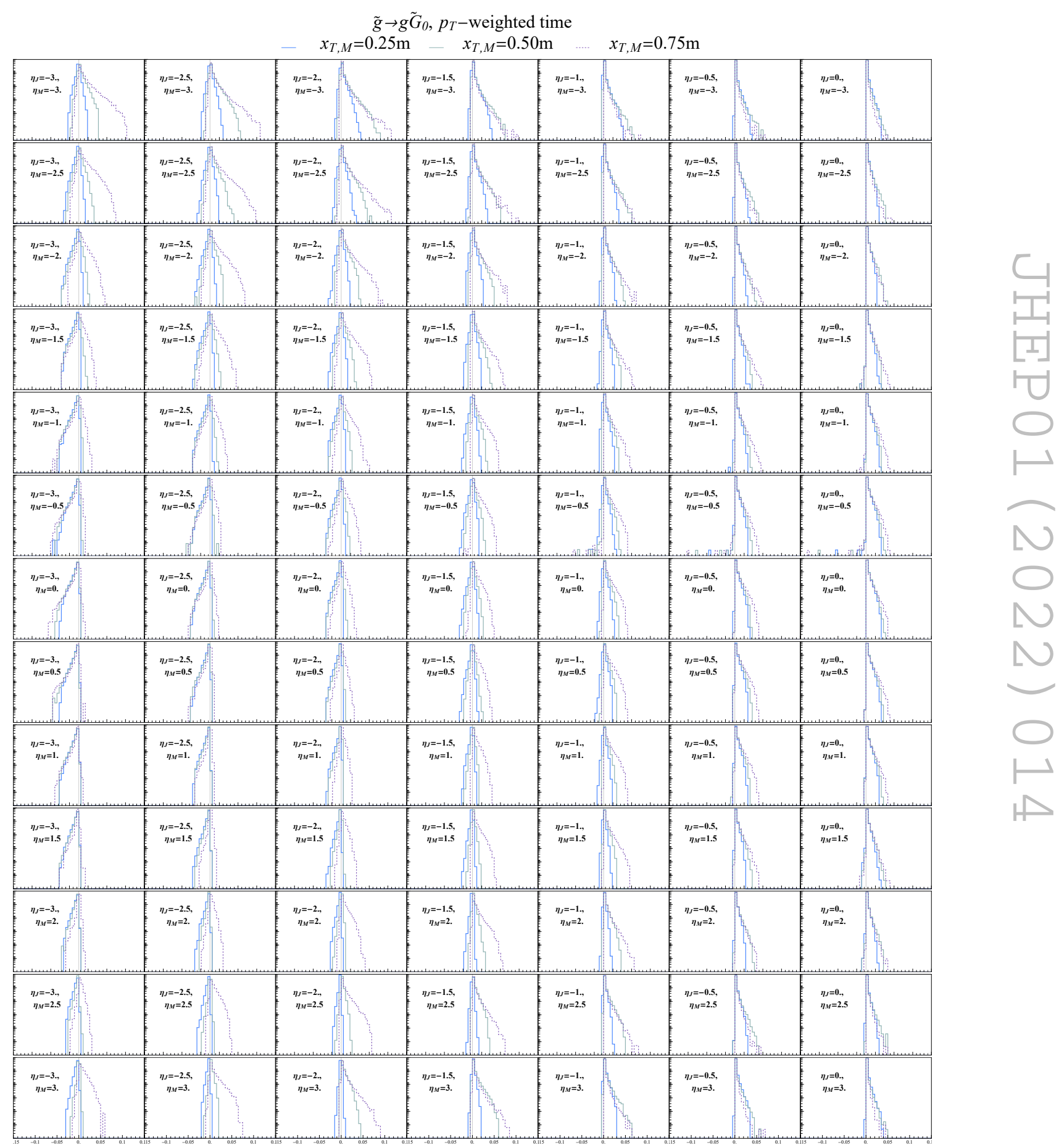

Figure 21. The relative time difference distribution for $x_{T, M}=0.25 \mathrm{~m}$ (blue), $x_{T, M}=0.50 \mathrm{~m}$ (gray), and $x_{T, M}=0.75 \mathrm{~m}$ (purple dashed) times as a function of $\eta_{J}$ ( $x$-axis) and of $\eta_{M}$ (y-axis) for the $p_{T}$-weighted time. The vertical axis in each plot is in log-scale and ranges from $10^{-5}$ to 1 . 


\section{References}

[1] CMS collaboration, Search for long-lived charged particles in proton-proton collisions at $\sqrt{s}=13$ TeV, Phys. Rev. D 94 (2016) 112004 [arXiv:1609.08382] [InSPIRE].

[2] ATLAS collaboration, Search for heavy charged long-lived particles in the ATLAS detector in $36.1 \mathrm{fb}^{-1}$ of proton-proton collision data at $\sqrt{\mathrm{s}}=13 \mathrm{TeV}$, Phys. Rev. D 99 (2019) 092007 [arXiv: 1902.01636] [INSPIRE].

[3] CMS collaboration, Search for decays of stopped exotic long-lived particles produced in proton-proton collisions at $\sqrt{s}=13 \mathrm{TeV}$, JHEP 05 (2018) 127 [arXiv:1801.00359] [INSPIRE].

[4] ATLAS collaboration, A search for the decays of stopped long-lived particles at $\sqrt{s}=13 \mathrm{TeV}$ with the ATLAS detector, JHEP 07 (2021) 173 [arXiv:2104.03050] [INSPIRE].

[5] ATLAS collaboration, Search for nonpointing and delayed photons in the diphoton and missing transverse momentum final state in $8 \mathrm{TeV}$ pp collisions at the LHC using the ATLAS detector, Phys. Rev. D 90 (2014) 112005 [arXiv:1409.5542] [INSPIRE].

[6] CMS collaboration, Search for long-lived particles using delayed photons in proton-proton collisions at $\sqrt{s}=13 \mathrm{TeV}$, Phys. Rev. D 100 (2019) 112003 [arXiv:1909.06166] [InSPIRE].

[7] J. Liu, Z. Liu and L.-T. Wang, Enhancing long-lived particles searches at the LHC with precision timing information, Phys. Rev. Lett. 122 (2019) 131801 [arXiv:1805.05957] [INSPIRE].

[8] M.D. Klimek, The time substructure of jets and boosted object tagging, arXiv:1911.11235 [INSPIRE].

[9] Z. Flowers, Q. Meier, C. Rogan, D.W. Kang and S.C. Park, Timing information at HL-LHC: complete determination of masses of dark matter and long lived particle, JHEP 03 (2020) 132 [arXiv:1903.05825] [INSPIRE].

[10] Z. Flowers, Q. Meier, C. Rogan, D.W. Kang and S.C. Park, Timing information at HL-LHC: complete determination of masses of dark matter and long lived particle, JHEP 03 (2020) 132 [arXiv: 1903.05825] [INSPIRE].

[11] S. Banerjee, B. Bhattacherjee, A. Goudelis, B. Herrmann, D. Sengupta and R. Sengupta, Determining the lifetime of long-lived particles at the HL-LHC, Eur. Phys. J. C 81 (2021) 172 [arXiv: 1912.06669] [INSPIRE].

[12] K.J. Bae, M. Park and M. Zhang, Demystifying freeze-in dark matter at the LHC, Phys. Rev. D 101 (2020) 115036 [arXiv:2001.02142] [INSPIRE].

[13] S. El Hedri and M. de Vries, Cornering colored coannihilation, JHEP 10 (2018) 102 [arXiv: 1806.03325] [INSPIRE].

[14] O. Cerri, S. Xie, C. Pena and M. Spiropulu, Identification of long-lived charged particles using time-of-flight systems at the upgraded LHC detectors, JHEP 04 (2019) 037 [arXiv: 1807.05453] [INSPIRE].

[15] A. Abada, N. Bernal, M. Losada and X. Marcano, Inclusive displaced vertex searches for heavy neutral leptons at the LHC, JHEP 01 (2019) 093 [arXiv:1807.10024] [INSPIRE].

[16] C. Frugiuele, E. Fuchs, G. Perez and M. Schlaffer, Relaxion and light (pseudo)scalars at the HL-LHC and lepton colliders, JHEP 10 (2018) 151 [arXiv:1807.10842] [INSPIRE]. 
[17] G.D. Kribs, A. Martin, B. Ostdiek and T. Tong, Dark mesons at the LHC, JHEP 07 (2019) 133 [arXiv: 1809.10184] [INSPIRE].

[18] A. Berlin and F. Kling, Inelastic dark matter at the LHC lifetime frontier: ATLAS, CMS, LHCb, CODEX-b, FASER, and MATHUSLA, Phys. Rev. D 99 (2019) 015021 [arXiv: 1810.01879] [INSPIRE].

[19] L.-X. Xu, J.-H. Yu and S.-H. Zhu, Minimal neutral naturalness model, Phys. Rev. D 101 (2020) 095014 [arXiv:1810.01882] [INSPIRE].

[20] G. Bélanger et al., LHC-friendly minimal freeze-in models, JHEP 02 (2019) 186 [arXiv: 1811.05478] [INSPIRE].

[21] J.A. Evans and M.A. Luty, Stopping quirks at the LHC, JHEP 06 (2019) 090 [arXiv: 1811.08903] [INSPIRE].

[22] C. Kilic, S. Najjari and C.B. Verhaaren, Discovering the twin Higgs boson with displaced decays, Phys. Rev. D 99 (2019) 075029 [arXiv:1812.08173] [InSPIRE].

[23] A. Delgado, A. Martin and M. Quirós, Higgsino dark matter in an economical Scherk-Schwarz setup, Phys. Rev. D 99 (2019) 075015 [arXiv: 1812.08019] [InSPIRE].

[24] J. Liu, Z. Liu, L.-T. Wang and X.-P. Wang, Seeking for sterile neutrinos with displaced leptons at the LHC, JHEP 07 (2019) 159 [arXiv:1904.01020] [INSPIRE].

[25] S. Chakraborti, V. Martin and P. Poulose, Freeze-in and freeze-out of dark matter with charged long-lived partners, JCAP 03 (2020) 057 [arXiv: 1904.09945] [INSPIRE].

[26] J. Serra, S. Stelzl, R. Torre and A. Weiler, Hypercharged naturalness, JHEP 10 (2019) 060 [arXiv: 1905.02203] [INSPIRE].

[27] J.D. Mason, Time-delayed electrons from Higgs decays to right-handed neutrinos, JHEP 07 (2019) 089 [arXiv: 1905.07772] [INSPIRE].

[28] M. Du, Z. Liu and V.Q. Tran, Enhanced long-lived dark photon signals at the LHC, JHEP 05 (2020) 055 [arXiv: 1912.00422] [INSPIRE].

[29] CMS collaboration, The phase-2 upgrade of the CMS level-1 trigger, Tech. Rep. CERN-LHCC-2020-004, CERN, Geneva, Switzerland (2020).

[30] B. Shuve and D. Tucker-Smith, Baryogenesis and dark matter from freeze-in, Phys. Rev. D 101 (2020) 115023 [arXiv:2004.00636] [INSPIRE].

[31] C. Yuan, H. Zhang, Y. Zhao and G. Chen, Producing and detecting long-lived particles at different experiments at the LHC, arXiv:2004.08820 [INSPIRE].

[32] J. Liu, Z. Liu, L.-T. Wang and X.-P. Wang, Enhancing sensitivities to long-lived particles with high granularity calorimeters at the LHC, JHEP 11 (2020) 066 [arXiv:2005.10836] [INSPIRE].

[33] E. Fuchs, O. Matsedonskyi, I. Savoray and M. Schlaffer, Collider searches for scalar singlets across lifetimes, JHEP 04 (2021) 019 [arXiv : 2008.12773] [INSPIRE].

[34] Y. Gershtein, S. Knapen and D. Redigolo, Probing naturally light singlets with a displaced vertex trigger, Phys. Lett. B $\mathbf{8 2 3}$ (2021) 136758 [arXiv:2012.07864] [INSPIRE].

[35] M. Borsato et al., Unleashing the full power of LHCb to probe stealth new physics, arXiv: 2105.12668 [INSPIRE]. 
[36] K. Cheung, K. Wang and Z.S. Wang, Time-delayed electrons from neutral currents at the LHC, JHEP 09 (2021) 026 [arXiv:2107.03203] [INSPIRE].

[37] K.R. Dienes, D. Kim, T. Leininger and B. Thomas, Tumblers: a novel collider signature for long-lived particles, arXiv:2108.02204 [INSPIRE].

[38] B. Bhattacherjee, S. Mukherjee, R. Sengupta and P. Solanki, Triggering long-lived particles in HL-LHC and the challenges in the RST stage of the trigger system, JHEP 08 (2020) 141 [arXiv: 2003.03943] [INSPIRE].

[39] C. Allaire et al., Beam test measurements of low gain avalanche detector single pads and arrays for the ATLAS high granularity timing detector, 2018 JINST 13 P06017 [arXiv: 1804.00622] [INSPIRE].

[40] CMS collaboration, Technical proposal for a MIP timing detector in the CMS experiment phase 2 upgrade, Tech. Rep. CERN-LHCC-2017-027, CERN, Geneva, Switzerland (2017).

[41] LHCb collaboration, Expression of interest for a phase-II LHCb upgrade: opportunities in flavour physics, and beyond, in the HL-LHC era, Tech. Rep. CERN-LHCC-2017-003, CERN, Geneva, Switzerland (2017).

[42] CMS collaboration, Search for long-lived particles using nonprompt jets and missing transverse momentum with proton-proton collisions at $\sqrt{s}=13$ TeV, Phys. Lett. B 797 (2019) 134876 [arXiv: 1906.06441] [INSPIRE].

[43] G.P. Salam, Towards jetography, Eur. Phys. J. C 67 (2010) 637 [arXiv:0906.1833] [INSPIRE].

[44] CMS collaboration, CMS tracking POG performance plots for 2017 with phase I pixel detector, https://twiki.cern.ch/twiki/bin/view/CMSPublic/TrackingPOGPerformance2017MC, (2017).

[45] M. Cacciari, G.P. Salam and G. Soyez, The catchment area of jets, JHEP 04 (2008) 005 [arXiv: 0802.1188] [INSPIRE].

[46] D. Krohn, J. Thaler and L.-T. Wang, Jets with variable R, JHEP 06 (2009) 059 [arXiv: 0903.0392] [INSPIRE].

[47] T. Sjöstrand et al., An introduction to PYTHIA 8.2, Comput. Phys. Commun. 191 (2015) 159 [arXiv: 1410.3012] [INSPIRE].

[48] M. Cacciari, G.P. Salam and G. Soyez, The anti- $k_{t}$ jet clustering algorithm, JHEP 04 (2008) 063 [arXiv:0802.1189] [INSPIRE].

[49] M. Cacciari, G.P. Salam and G. Soyez, FastJet user manual, Eur. Phys. J. C 72 (2012) 1896 [arXiv: 1111.6097] [INSPIRE].

[50] J. Alwall et al., The automated computation of tree-level and next-to-leading order differential cross sections, and their matching to parton shower simulations, JHEP 07 (2014) 079 [arXiv: 1405.0301] [INSPIRE].

[51] CMS collaboration, CMS, the Compact Muon Solenoid: technical proposal, Tech. Proposal, CERN, Geneva, Switzerland (1994).

[52] ATLAS collaboration, ATLAS: technical proposal for a general-purpose pp experiment at the Large Hadron Collider at CERN, Tech. Proposal, CERN, Geneva, Switzerland (1994). 
[53] CMS collaboration, A MIP timing detector for the CMS phase-2 upgrade, Tech. Rep. CERN-LHCC-2019-003, CERN, Geneva, Switzerland (2019).

[54] CMS collaboration, Particle-flow reconstruction and global event description with the CMS detector, 2017 JINST 12 P10003 [arXiv:1706.04965] [INSPIRE].

[55] D. Krohn, J. Thaler and L.-T. Wang, Jet trimming, JHEP 02 (2010) 084 [arXiv:0912.1342] [INSPIRE].

[56] D. Krohn, M.D. Schwartz, M. Low and L.-T. Wang, Jet cleansing: pileup removal at high luminosity, Phys. Rev. D 90 (2014) 065020 [arXiv: 1309.4777] [INSPIRE].

[57] P. Berta, M. Spousta, D.W. Miller and R. Leitner, Particle-level pileup subtraction for jets and jet shapes, JHEP 06 (2014) 092 [arXiv: 1403.3108] [INSPIRE].

[58] D. Bertolini, P. Harris, M. Low and N. Tran, Pileup per particle identification, JHEP 10 (2014) 059 [arXiv: 1407.6013] [INSPIRE].

[59] M. Cacciari, G.P. Salam and G. Soyez, SoftKiller, a particle-level pileup removal method, Eur. Phys. J. C 75 (2015) 59 [arXiv: 1407.0408] [InSPIRE].

[60] P.T. Komiske, E.M. Metodiev, B. Nachman and M.D. Schwartz, Pileup mitigation with machine learning (PUMML), JHEP 12 (2017) 051 [arXiv:1707.08600] [INSPIRE]. 